\title{
Facets for the cut cone II
}

Deza, M.M.; Laurent, Monique

Published in:

Mathematical Programming

Publication date:

1992

Link to publication

Citation for published version (APA):

Deza, M. M., \& Laurent, M. (1992). Facets for the cut cone II: Clique-web inequalities. Mathematical Programming, 56(1-3), 161-188.

\section{General rights}

Copyright and moral rights for the publications made accessible in the public portal are retained by the authors and/or other copyright owners and it is a condition of accessing publications that users recognise and abide by the legal requirements associated with these rights.

- Users may download and print one copy of any publication from the public portal for the purpose of private study or research

- You may not further distribute the material or use it for any profit-making activity or commercial gain

- You may freely distribute the URL identifying the publication in the public portal

Take down policy

If you believe that this document breaches copyright, please contact us providing details, and we will remove access to the work immediately and investigate your claim. 


\title{
Facets for the cut cone II: Clique-web inequalities
}

\section{Michel Deza}

CNRS, Université Paris VII, UA 212, 75251 Paris 05, France

\section{Monique Laurent}

CNRS, LAMSADE, Université Paris Dauphine, 75775 Paris 16, France

Received 31 May 1989

Revised manuscript received 5 March 1991

\begin{abstract}
We study new classes of facets for the cut cone $C_{n}$ generated by the cuts of the complete graph on $n$ vertices. This cone can also be interpreted as the cone of all semi-metrics on $n$ points that are isometrically $l_{1}$-embeddable and, in fact, the study of the facets of the cut polytope is in some sense equivalent to the study of the facets of $C_{n}$. These new facets belong to the class of clique-web inequalities which generalize the hypermetric and cycle inequalities as well as the bicycle odd wheel inequalities.
\end{abstract}

Key words: Cone, polytope, facet, antiweb, cut, hypermetric inequality.

\section{Introduction}

This paper is a follow-up to [16] dealing with valid inequalities and facets of the cut cone $C_{n}$. The cut cone $C_{n}$ is the cone generated by the cuts of the complete graph $K_{n}$ on $n$ vertices. The cut polytope $P_{n}$ is the polytope whose vertices are the cuts of $K_{n}$. There are several motivations for the study of the cut cone. First, the study of the facial structure of the cut cone is relevant to the polyhedral approach to the max-cut problem which is a notorious NP-hard problem. The max-cut problem can be formulated as an optimization problem over the cut polytope, but a remarkable property of the cut polytope ([7]) is that all its facets can be deduced from the facets of the cut cone via a "switching" property, that we shall recall below. On the other hand, the cut cone $C_{n}$ is also relevant to the theory of finite metric spaces; namely, the elements of $C_{n}$ can be interpreted as the semi-metrics on $n$ points which are isometrically $l_{1}$-embeddable or, in other words, a vector $d$ of $\mathbb{R}^{n(n-1) / 2}$ belongs to $C_{n}$ if and only if there exist some vectors $x_{1}, \ldots, x_{n}$ in $\mathbb{R}^{m}$, for some $m \geqslant 1$, such that $d_{i j}=\left\|x_{i}-x_{j}\right\|_{1}$ for $1 \leqslant i<j \leqslant n$ (recall that $\|x\|_{1}=\sum_{1 \leqslant u \leqslant m}\left|x_{u}\right|$ for $x \in \mathbb{R}^{m}$ ). We refer e.g., to $[2,3]$ for more information on this connection. 
In [16], we introduced, in particular, cycle inequalities and briefly announced their generalization to clique-web inequalities ( $\mathrm{CW}$ inequalities, for short), This paper is devoted to the study of clique-web inequalities and is organized as follows.

In the first section, we introduce CW inequalities (in their pure and collapsed form) and we give a description of their roots, i.e., of the cuts realizing equality. In the second section, we give several new classes of facets of $C_{n}$ arising from $\mathrm{CW}$ inequalities. We also show that the pure $\mathrm{CW}$ inequalities yield facets of the equicut polytope. We group in Section 3 the proofs for the results on CW facets stated in Section 2.

In [15], we generalize CW inequalities for multicut polytopes.

We now give all notation and preliminaries needed for the paper. Our graph notation is classical, as well as the notions of polyhedral combinatorics that we will use. All graphs are simple and undirected. We denote by $[1, n]$ the set of the $n$ integers $1,2, \ldots, n . K_{n}$ denotes the complete graph on the $n$ nodes $1,2, \ldots, n$. Given a subset $S$ of $[1, n]$, the set $\delta(S)$ of all the edges of $K_{n}$ having exactly one endnode in $S$ is called the cut determined by $S$. Then, the incidence vector of the cut $\delta(S)$ is the vector $\chi^{\delta(S)}$, called the cut vector determined by $S$, and defined by $\chi_{i j}^{\delta(S)}=1$ if $i j$ is an edge of $\delta(S)$, i.e., $|S \cap\{i, j\}|=1$, and $\chi_{i j}^{\delta(S)}=0$ otherwise, for $1 \leqslant i<j \leqslant n$. Since $\delta(S)=\delta([1, n]-S)$, there are $2^{n-1}-1$ nonzero cut vectors. The cut cone $C_{n}$ is the cone generated by all cut vectors of $K_{n}$. The cone $C_{n}$ is a full dimensional polyhedral cone in $\mathbb{R}^{n(n-1) / 2}$ containing the origin.

Given a vector $v$ of $\mathbb{R}^{n(n-1) / 2}$, the inequality $v \cdot x \leqslant 0$ is called valid for $C_{n}$ if it is satisfied by all vectors of $C_{n}$, or, equivalently, by all cut vectors. Then, the set $F_{v}=\left\{x \in C_{n}: v \cdot x=0\right\}$ is the face generated by the valid inequality $v \cdot x \leqslant 0$, or simply by $v$. For a cut $\delta(S)$, we set $v(\delta(S))=\sum_{i j \in \delta(S)} v_{i j}$. The cuts $\delta(S)$ whose incidence vectors belong to $F_{v}$, i.e., the cuts $\delta(S)$ satisfying $v(\delta(S))=0$, are called the roots of $v$; we also say, for short, that the set $S$ itself, defines a root of $v$. The set of roots of $v$ is denoted by $R(v)$. The dimension of the face $F_{v}$ is the maximum number of affinely independent points in $F_{v}$ minus one, or, equivalently, since $F_{v}$ contains the origin, it is the maximum number of roots of $v$ whose incidence vectors are linearly independent. A facet is a maximal face of $C_{n}$, i.e., a face of dimension $\frac{1}{2} n(n-1)-1$; if $F_{v}$ is a facet, one also says that $v$ is facet inducing.

Given a vector $v$ of $\mathbb{R}^{n(n-1) / 2}$, its supporting graph $G(v)$ is the weighted graph with nodeset $[1, n]$ and whose edges are the pairs $(i, j)$ for which $v_{i j} \neq 0$, the edge $i j$ being assigned weight $v_{i j}$. Conversely, if $G$ is a (edge) weighted graph on $n$ nodes, its edgeweight vector is the vector $v$ of length $\frac{1}{2} n(n-1)$ where, for the edges $i j$ of $G, v_{i j}$ is the weight of edge $i j$ and $v_{i j}=0$ if $i j$ is not an edge of $G$. If $G$ is a graph, then, when the weights are not specified, they are assumed to be the unit weights, i.e, we have $v_{i j}=1$ if $i j$ is an edge of $G$ and $v_{i j}=0$ otherwise. We call an inequality $v \cdot x \leqslant 0$ pure if the components $v_{i j}$ of $v$ take only values $+1,-1,0$.

Known classes of valid inequalities for the cone $C_{n}$ include hypermetric inequalities introduced in [10] and later independently in [18] and cycle inequalities introduced in [16]. Let $b_{1}, b_{2}, \ldots, b_{n}$ be integers such that $b_{1}+\cdots+b_{n}=1$ and set 
$b=\left(b_{1}, \ldots, b_{n}\right)$; then, the hypermetric inequality $\operatorname{Hyp}_{n}(b)$ is the inequality defined by

$$
\operatorname{Hyp}_{n}(b) \cdot x=\sum_{1 \leqslant i<j \leqslant n} b_{i} b_{j} x_{i j} \leqslant 0 .
$$

Let $b_{1}, \ldots, b_{n}$ be integers such that $b_{1}+\cdots+b_{n}=3, b_{1}, \ldots, b_{p}>0 \geqslant b_{p+1}, \ldots, b_{n}$ and set $b=\left(b_{1}, \ldots, b_{n}\right)$; then the cycle inequality $\mathrm{Cyc}_{n}(b)$ is the inequality defined by

$$
\operatorname{Cyc}_{n}(b) \cdot x=\sum_{1 \leqslant i<j \leqslant n} b_{i} b_{j} x_{i j}-\left(\sum_{I \leqslant i \leqslant p-1} x_{i, i+1}+x_{i p}\right) \leqslant 0 .
$$

In other words, the second term in $\mathrm{Cyc}_{n}(b) \cdot x$ is equal to $\sum_{i j \in C} x_{i j}$ where $C=$ $(1,2, \ldots, p)$ is the cycle with nodes $1, \ldots, p$ and edges $(i, i+1)$ for $1 \leqslant i \leqslant p$ (the indices being taken modulo $p$ ). When all (resp. all except at most one) negative components of $b=\left(b_{1}, \ldots, b_{n}\right)$ are equal to -1 , we say that $b$ is linear (resp. quasilinear); we correspondingly define linear and quasilinear hypermetric and cycle inequalities.

Given a vector $v$ of $\mathbb{R}^{n(n-1) / 2}$ and a cut $\delta(S)$ of $K_{n}$, consider the vector $v^{s}$ of $\mathbb{R}^{n(n-1) / 2}$ defined by $v_{i j}^{s}=-v_{i j}$ if $i j$ is an edge of the cut $\delta(S)$ and $v_{i j}^{s}=v_{i j}$ otherwise; one says that $v^{s}$ is obtained by switching of $v$ by the cut $\delta(S)$. Let $P_{n}$ denote the cut polytope of the complete graph $K_{n}$, i.e., $P_{n}$ is the convex hull of all cut vectors. If $v \cdot x \leqslant v_{0}$ is a valid inequality for $P_{n}$, then $v^{5} \cdot x \leqslant v_{0}-v(\delta(S))$ is also a valid inequality for $P_{n}$ and $v \cdot x \leqslant v_{0}$ is facet inducing for $P_{n}$ if and only if $v^{5} \cdot x \leqslant$ $v_{0}-v(\delta(S))$ is facet inducing for $P_{n}$ [7]; one then says that both facets are switching equivalent. Therefore, all facets of $P_{n}$ can be obtained by switching by cuts of the facets of $C_{n}$. This connection between the cut cone and the cut polytope remains valid for the general case of non complete graphs [7]; the switching operation on facets of $C_{n}$ was introduced in [11]. See [13] for a more detailed information on the symmetries of the cut polytope $P_{n}$.

Finally, recall that zero-lifting preserves facets of the cut cone. Namely, for $v \in \mathbb{R}^{n(n-1) / 2}$, define $v^{\prime} \in \mathbb{R}^{n(n+1) / 2}$ by $v_{i j}^{\prime}=v_{i j}$ if $1 \leqslant i<j \leqslant n$ and $v_{i n+1}^{\prime}=0$ if $1 \leqslant i \leqslant n$; then, the inequality $v \cdot x \leqslant 0$ defines a facet of $C_{n}$ if and only if the inequality $v^{\prime} \cdot x \leqslant 0$ defines a facet of $C_{n+1}[11,16]$. Therefore, when we have an inequality whose supporting graph spans $n$ nodes, if we can show that it defines a facet of the cone $C_{n}$, then it also defines a facet of the cone $C_{m}$ for any $m \geqslant n$.

\section{Clique-web inequalities}

\subsection{Pure $C W$ inequalities $C W_{n}^{k}$}

Let $n, p, q, k$ be nonnegative integers satisfying

$$
n=p+q, \quad p-q=2 k+1, \quad q \geqslant 2,
$$


or, equivalently

$$
p=\frac{1}{2}(n+1)+k, \quad q=\frac{1}{2}(n-1)-k, \quad 0 \leqslant k \leqslant \frac{1}{2}(n-5) .
$$

Let $\mathrm{AW}_{p}^{k}$ denote the antiweb with parameters $p, k$, i.e., $\mathrm{AW}_{p}^{k}$ is the graph with nodeset $[1, p]$ and edges $(i, i+1),(i, i+2), \ldots,(i, i+k)$ for $i \in[1, p]$ (the indices being taken modulo $p$ ); the complement of $\mathrm{AW}_{p}^{k}$ is the web $W_{p}^{k}$. Webs and antiwebs are Cayley graphs on the group $Z_{p}$. In the following, we use the same notation $A W_{p}^{k}$ for denoting the antiweb graph and its set of edges.

Definition 1.1. The pure clique-web inequality ( $\mathrm{CW}$ inequality, for short) $\mathrm{CW}_{n}^{k}$ with parameters $n, p, q, k$ satisfying (1.1) is the inequality

$$
\mathrm{CW}_{n}^{k} \cdot x=\sum_{1 \leq i<j \leqslant n} b_{i} b_{j} x_{i j}-\sum_{i j \in \mathrm{AW}_{p}^{k}} x_{i j} \leqslant 0,
$$

with $b=(1, \ldots, 1,-1, \ldots,-1)$ whose first $p$ coefficients are +1 and last $q=n-p$ coefficients are -1 .

Remark 1.2. We have chosen the terminology "clique-web" inequality, since (1.3) can also be written as

$$
\sum_{i j \in W_{p}^{k}} x_{i j}+\sum_{p+1 \leqslant i<j \leqslant n} x_{i j}-\sum_{1 \leqslant i \leqslant p, p+1 \leqslant j \leqslant n} x_{i j} \leqslant 0 .
$$

Hence, there is a web on the first $p$ nodes (the nodes for which $b_{i}=+1$ ) and a clique on the remaining $q=n-p$ nodes (the nodes for which $b_{i}=-1$ ). We shall denote in the remainder of the paper the $q$ "negative" nodes $p+1, \ldots, n$ (i.e., those for which $b_{i}=-1$ ) by $1^{\prime}, \ldots, q^{\prime}$.

Note that, if we relax the equality condition $p-q=2 k+1$ from (1.1) to the condition $p-q \geqslant 2 k+1$, then (1.3) is no more valid for $C_{n}$; however, a suitable positive value for the right hand side of (1.3), namely, the value $\frac{1}{2}(p-q) \times$ $(p-q-2 k-1)$, restores validity and actually gives validity for general multicut polytopes (see [15]).

As an example, Figure 1 shows the supporting graph of the $\mathrm{CW}_{11}^{2}$ inequality (edges with weight +1 are indicated by a plain line while edges with weight -1 are indicated by a dotted line and every node of the triangle is joined to every node of the web).

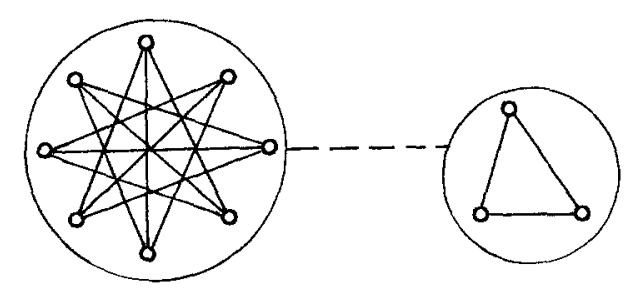

Fig. 1. 
Remark 1.3. We restricted our attention to the case $q \geqslant 2$, because inequality (1.3) for $q=1$ takes the following form:

$$
-\sum_{1 \leqslant j \leqslant n-1} x_{n j}+\sum_{1 \leqslant i \leqslant k+1} x_{i, k+i+1}=\sum_{1 \leqslant i \leqslant k+1}\left(x_{i, k+i+1}-x_{n i}-x_{n, k+i+1}\right) \leqslant 0,
$$

and, therefore, is the sum of $k+1$ triangle inequalities, i.e., except for $k=0$, it is not facet inducing.

For $k=0, \mathrm{AW}_{p}^{0}$ is just the empty graph and the $\mathrm{CW}_{n}^{0}$ inequality is the pure hypermetric inequality $\operatorname{Hyp}_{n}(1, \ldots, 1,-1, \ldots,-1)$ (cf. $\left.[10,18,16]\right)$. In the other extreme case: $k=\frac{1}{2}(n-5)$, the web $W_{p}^{k}(p=2 k+3)$ is a cycle and the inequality (1.3) corresponds (up to switching) to the bicycle odd wheel inequality given in [7] as facet for the cut polytope (and in [6] as facet for the bipartite subgraph polytope); it is the $\mathrm{CW}_{n}^{k}$ inequality with $n=2 k+5$, i.e., $p=2 k+3$ and $q=2$. In the case $k=1$, $\mathrm{AW}_{p}^{1}$ is a cycle and the inequality (1.3) is exactly the pure cycle inequality $\operatorname{Cyc}_{n}(1, \ldots, 1,-1, \ldots,-1)$ introduced in [16]. It is actually the inspection of the above three cases which led us to the definition of $\mathrm{CW}$ inequalities.

Actually, hypermetric inequalities $\operatorname{Hyp}_{n}(b)$ and cycle inequalities $\mathrm{Cyc}_{n}(b)$ are defined, more generally, for any integers $b_{1}, \ldots, b_{n}$ with $b_{1}+\cdots+b_{n}=1$ or 3 , respectively. In the next section, we define general $\mathrm{CW}$ inequalities $\mathrm{CW}_{n}^{k}(b)$ for any integers $b_{1}, \ldots, b_{n}$ with $b_{1}+\cdots+b_{n}=2 k+1, k \geqslant 0$. For this, we need the concept of collapsing applied to antiwebs.

\subsection{General $C W$ inequality $C W_{n}^{k}(b)$}

Given integers $h \geqslant p \geqslant 1$, take a partition $\pi$ of the set $[1, h]$ into $p$ parts $I_{1}, \ldots, I_{p}$, $[1, h]=\bigcup_{i \in[1, p]} I_{i}$. If $v$ is a vector of length $\frac{1}{2} h(h-1)$, its $\pi$-collapse is the vector $v_{\pi}$ of length $\frac{1}{2} p(p-1)$ defined by $\left(v_{\pi}\right)_{i j}=\sum_{a \in I_{i}, b \in I_{i}} v_{a b}$ for all $1 \leqslant i<j \leqslant p$.

If $G$ is a (edge) weighted graph on nodeset $[1, h]$ and if $v$ denotes its edgeweight vector, then the $\pi$-collapse of $G$ is the weighted graph $G_{\pi}$ on nodeset $[1, p]$ whose edgeweight vector is $v_{\pi}$. In other words one obtains $G_{\pi}$ from $G$ by contracting all nodes from a common partition class into a single node and correspondingly adding the edge weights.

Given a subset $S$ of $[1, p]$, set $S^{\pi}=\bigcup_{i \in S} I_{i}$; so $S^{\pi}$ is a subset of $[1, h]$ and one checks easily that

$$
v_{\pi}(\delta(S))=v\left(\delta\left(S^{\pi}\right)\right) \quad \text { for all } S \subseteq[1, p] .
$$

In consequence, if $v \cdot x \leqslant 0$ defines a valid inequality of the cone $C_{h}$ (defined on the $h$ nodes $1,2, \ldots, h)$, then its $\pi$-collapse $v_{\pi} \cdot x \leqslant 0$ defines a valid inequality of the cone $C_{p}$ (defined on the $p$ nodes $1,2, \ldots, p$ ). Furthermore, the set of roots of $v_{\pi} \cdot x \leqslant 0$ is given by

$$
R\left(v_{\pi}\right)=\left\{\delta(S): S \subseteq[1, p] \text { and } \delta\left(S^{\pi}\right) \in R(v)\right\} .
$$


Hence, collapsing is an operation that preserves validity and so it is a useful tool for producing large new classes of valid inequalities (see [9] for a general study of the collapsing operation for the cut cone). Actually, we checked that most of the known facets of $C_{n}$ are pure or collapses of some pure facets. We conjecture that any non pure facet of $C_{n}$ is the collapse of some pure facet. Note that collapsing does not always preserve facethood, also it may be that the collapse of a non facet inducing inequality be facet inducing. For example, the inequality: $\left(x_{23}-x_{12}-x_{13}\right)+$ $\left(x_{45}-x_{14}-x_{15}\right) \leqslant 0$ is not facet inducing for $C_{5}$ (it is the sum of two triangle inequalities), while the inequality obtained by collapsing both nodes 1,5 into the node 1: $x_{23}-x_{12}-x_{13} \leqslant 0$ is indeed facet inducing.

Let us now define a class of weighted antiwebs; they are constructed by collapsing the antiwebs, but we use a specific partition for the collapsing operation. Let $b_{1}, \ldots, b_{p}$ be positive integers, set $b=\left(b_{1}, \ldots, b_{p}\right)$ and $h=b_{1}+\cdots+b_{p}$. Consider the partition $\pi_{0}(b)$ of $[1, h]$ consisting of the $p$ intervals $I_{0}=\left[1, b_{1}\right]$ and $I_{i}=$ $\left[b_{1}+\cdots+b_{i}+1, b_{1}+\cdots+b_{i}+b_{i+1}\right]$ for $i=1, \ldots, p-1$.

Definition 1.4. With the above notation, the antiweb $\mathrm{AW}_{p}^{k}(b)$ is the weighted graph obtained by $\pi_{0}(b)$-collapsing the antiweb $\mathrm{AW}_{h}^{k}$.

Note that, in the above definition, we consider interval-collapsing, i.e., collapsing using an interval partition of the circularly ordered nodeset $[1, h]$ into $p$ consecutive intervals. Clearly, $\mathrm{AW}_{p}^{k}(1, \ldots, 1)$ is just the usual antiweb $\mathrm{AW}_{p}^{k}$ but, for arbitrary $b, \mathrm{AW}_{p}^{k}(b)$ might be a rather complicated weighted graph. We give below two examples of collapsed antiwebs.

Example 1.5. If $b_{i} \geqslant k$ for all $i \in[1, p]$, then $\mathrm{AW}_{p}^{k}\left(b_{1}, \ldots, b_{p}\right)$ is $\frac{1}{2} k(k+1) \mathrm{AW}_{p}^{1}$, i.e., it is the cycle $C(1,2, \ldots, p)$ with weight $\frac{1}{2} k(k+1)$ on its edges. In particular, for $k=1$, interval-collapsing of a cycle is a cycle.

Example 1.6. $\mathrm{AW}_{p}^{k}(2,1, \ldots, 1)$ is the weighted graph obtained from $\mathrm{AW}_{p}^{k}$ by

- deleting the edges $(p-i, k-i)$ for $i=0,1, \ldots, k-2$,

- assigning weight 2 to the edges $(1, i)$ and $(1, p-k+i)$ for $i=2, \ldots, k$.

Figures 2(a) and 2(b) show respectively the antiweb $A_{6}^{2}$ and the collapsed antiweb $\mathrm{AW}_{4}^{2}(2,2,2,2)$ (see that $\mathrm{AW}_{4}^{2}(2,2,2,2)$ is obtained from $\mathrm{AW}_{8}^{2}$ by contracting

(a)

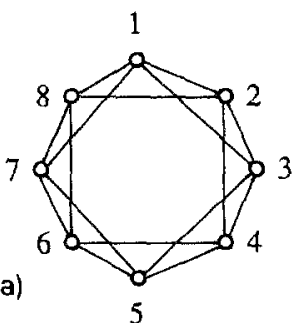

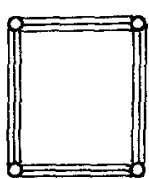

(b)

Fig. 2. 


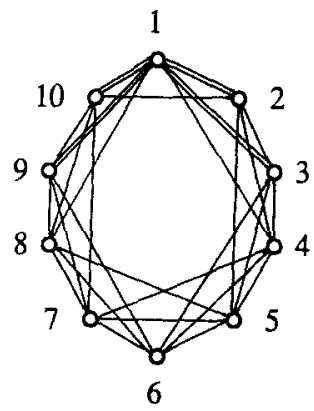

Fig. 3.

the two nodes 1 and 2 into a single node, similarly for the nodes 3 and 4, 5 and 6 , 7 and 8, and then adding the corresponding edgeweights). Figure 3 shows the antiweb $\mathrm{AW}_{10}^{3}(2,1,1,1,1,1,1,1,1,1)$. (Edges with weight 2 are indicated by a double line and edges with weight 3 by a triple line.)

We can now define general $\mathrm{CW}$ inequalities $\mathrm{CW}_{n}^{k}(b)$. Let $b=\left(b_{1}, \ldots, b_{n}\right)$ be integers whose sum is $b_{1}+\cdots+b_{n}=2 k+1(k \geqslant 0)$ and let $p$ (resp. q) denote the number of positive (resp. nonpositive) $b_{i}$ 's; so $n=p+q$. Let $B_{+}=\left\{i \in[1, n]: b_{i}>0\right\}$ denote the positive support of $b$, so $\left|B_{+}\right|=p$. We can suppose (w.l.o.g. for our purpose) that $B_{+}=[1, p]$, i.e., $b_{1}, \ldots, b_{p}>0$ (actually, permuting the coefficients $b_{i}$ in $C W_{n}^{k}(b)$ may affect facethood for $k \geqslant 1$, cf. Example 3.24 in [16] for $k=1$; while, for $k=0$, any permutation preserves facets). We consider the collapsed antiweb $\mathrm{AW}_{p}^{k}\left(b_{1}, \ldots, b_{p}\right)$, also denoted by $\mathrm{AW}_{p}^{k}(b)$, for short, defined on the nodeset $B_{+}=$ $[1, p]$.

Definition 1.7. With the above notation, the general $\mathrm{CW}$ inequality $\mathrm{CW}_{n}^{k}(b)$ is the inequality

$$
\mathrm{CW}_{n}^{k}(b) \cdot x=\sum_{1 \leqslant i<j \leqslant n} b_{i} b_{j} x_{i j}-\sum_{i j \in \mathrm{AW}_{p}^{k}(b)} x_{i j} \leqslant 0 .
$$

In Definition 1.7, relation $\sum_{i j \in A w_{f}^{\prime}(b)} x_{i j}$ should be understood as $\sum_{1 \leqslant i<j \leqslant p} v_{i j} x_{i j}$, where $v=\left(v_{i j}\right)_{\leqslant i<j \leqslant p}$ is the edgeweight vector of the weighted graph $\mathrm{AW}_{p}^{k}(b)$. Note that the general $\mathrm{CW}$ inequality $\mathrm{CW}_{n}^{k}(b)$ is, in fact, a collapsing of the pure $\mathrm{CW}$ inequality $\mathrm{CW}_{m}^{k}$, where $m=\sum_{1 \leqslant i \leqslant n}\left|b_{i}\right|$. Namely, consider the partition $\pi(b)$ of $[1, m]$ into the $p$ intervals $I_{0}, \ldots, I_{p-1}$ (having sizes $b_{1}, \ldots, b_{p}$ and forming the previously considered partition $\pi_{0}(b)$ of $\left.[1, h], h=\sum_{1 \leqslant i \leqslant p} b_{i}\right)$ and $q=n-p$ arbitrary parts (of sizes $\left|b_{p+1}\right|, \ldots,\left|b_{n}\right|$ and partitioning $[h+1, m]$ ); then, $\mathrm{CW}_{n}^{k}(b)$ is just the $\pi(b)$-collapse of $\mathrm{CW}_{m}^{k}$.

In order to visualize the general $\mathrm{CW}$ inequality $\mathrm{CW}_{n}^{k}(b)$, one needs the explicit description of the collapsed antiweb $\mathrm{AW}_{p}^{k}(b)$. For example, Figure 4 shows the supporting graph of the $\mathrm{CW}$ inequality $\mathrm{CW}_{7}^{2}(2,2,2,2,-1,-1,-1)$ (the numbers on the edges indicate the edge weights) (recall Figure 2(b) for the description of $\left.\mathrm{AW}_{4}^{2}(2,2,2,2)\right)$. 


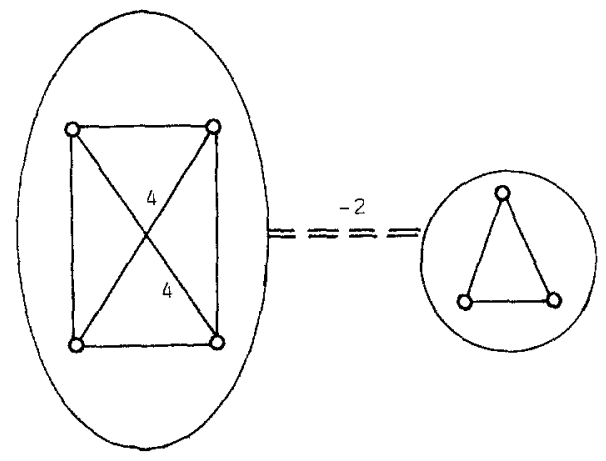

Fig. 4.

\subsection{Validity and roots}

\subsubsection{Validity}

For $k=0,1, \mathrm{CW}$ inequalities are the known valid hypermetric and cycle inequalities. We established validity of pure $\mathrm{CW}$ inequalities $\mathrm{CW}_{n}^{k}$ for the cases $k=2$ and $p>(k-1)\left(k^{2}+k-2\right)$ and conjectured the general result in [16]. We do not give the proof here, since Alon [1] proved validity of all pure $\mathrm{CW}$ inequalities. As mentioned in Section 1.2, the collapsing operation preserves validity; therefore, validity of general $\mathrm{CW}$ inequalities follows immediately from the pure case. The general $\mathrm{CW}$ inequality $\mathrm{CW}_{n}^{k}(b)$ can also be interpreted as arising from the corresponding pure inequality in which nodes are allowed to be repeated (the $b_{i}$ 's being the repetition numbers); in this form, validity of general $\mathrm{CW}$ inequalities was also noticed in [1]. But our way of defining general $\mathrm{CW}$ inequalities from pure ones via collapsing is necessary for obtaining explicit expression of collapsed antiweb, roots, etc. and, therefore, for studying CW facets.

\subsubsection{Roots of the pure $C W$ inequality}

For $k=0$, the roots of $\mathrm{CW}_{n}^{0}=\operatorname{Hyp}_{n}(1, \ldots, 1,-1, \ldots,-1)$ are evidently the cuts $\delta(S)$ with $S=S_{+} \cup S_{-}$where $S_{+}, S_{-}$are respectively subsets of $[1, p],\left[1^{\prime}, q^{\prime}\right]$ of sizes $s_{+}, s_{-}$with $s_{+}-s_{-}=0,1$. From now on we consider $\mathrm{CW}_{n}^{k}$ inequalities with $k \geqslant 1$.

Let $n=p+q$ with $p \geqslant 2 k+3, q \geqslant 2, p-q=2 k+1$; we denote the $n$ nodes by $[1, p] \cup\left[1^{\prime}, q^{\prime}\right]$ (as in Remark 1.2). We use the following fact; take a set $S=S_{+} \cup S_{-}$ with $S_{+} \subseteq[1, p]$ of size $s_{+}$and $S_{-} \subseteq\left[1^{\prime}, q^{\prime}\right]$ of size $s_{-}$, then $\delta(S)$ is a root of $\mathrm{CW}_{n}^{k}$ if and only if the following relation holds:

$$
\left(s_{+}-s_{-}\right)\left(2 k+1-\left(s_{+}-s_{-}\right)\right)=\left|\delta\left(S_{+}\right) \cap \mathrm{AW}_{p}^{k}\right| .
$$

Theorem 1.8. The roots of $\mathrm{CW}_{n}^{k}(n=p+q, k \geqslant 1)$ are the cuts $\delta(S)$ for which $S=S_{+} \cup$ $S_{-}$is of one of the following two types:

Type 1: $S_{-}=\emptyset$ and $S_{+}$indices a clique of the antiweb $\mathrm{AW}_{p}^{k}$, i.e., any two nodes of $S_{+}$are adjacent in $\mathrm{AW}_{p}^{k}$; 
Type 2: $S_{+}$is an interval of $[1, p]$ of size $s_{+}, k+1 \leqslant s_{+} \leqslant p-k$, and $S_{-}$is a subset of $\left[1^{\prime}, q^{\prime}\right]$ of size $s_{-}$, where $s_{+}-k-1 \leqslant s_{-} \leqslant s_{+}-k$.

Remark 1.9. When we say that a subset $S$ of $[1, p]$ is an interval, we mean, more precisely, that $S$ is a circular interval of the circularly ordered set $[1, p]$. There are some redundancies in the presentation of the roots given in Theorem 1.8; it is easy to see that a description of the roots in which each root occurs exactly once can be obtained by replacing the family of sets of Type 2 by the family of sets of Type $2^{\prime}$ where:

Type $2^{\prime}: S_{+}$is an interval of $[1, p]$ of size $s_{+}, k+1 \leqslant s_{+} \leqslant p-k-2$, and $s_{-}$is a subset of $\left[1^{\prime}, q^{\prime}\right]$ of size $s_{-}$, where $s_{-}=s_{+}-k$.

Remark 1.10. It is easy to see that, if $S \subseteq[1, p]$ induces a clique of the antiweb $\mathrm{AW}_{p}^{k}$, then $|S| \leqslant k+1$ holds. For example, the interval $[1, k+1]=\{1,2, \ldots, k, k+1\}$ induces a clique of $\mathrm{AW}_{p}^{k}$; therefore, any subset $S$ of the following Type $1^{*}$ induces a clique of $\mathrm{AW}_{p}^{k}$.

Type $1^{*}: S$ is contained in an interval of size $k+1$ of $[1, p]$.

There may exist other subsets of $[1, p]$ inducing cliques of $\mathrm{AW}_{p}^{k}$, for example, for $k=3, p=9$, the set $\{1,4,7\}$ induces a clique of $\mathrm{AW}_{9}^{3}$. In fact, there exist additional subsets of $[1, p]$ inducing cliques of $A W_{p}^{k}$ besides those of Type $1^{*}$ only for $p \leqslant 3 k$. Actually, all our proofs for $\mathrm{CW}$ facets use only the roots $\delta(S)$ for which $S$ is of Type $1^{*}$ or 2 .

In the remaining of this section, we give the proof of Theorem 1.8 through Claims $1.11,1.13,1.14,1.15$ and 1.16 .

Claim 1.11. If $S$ is of Type 1 or 2 , then $\delta(S)$ is indeed a root of $\mathrm{CW}_{n}^{k}$.

Proof. Take first $S$ of Type 1 with $s=|S|$. Since $S$ induces a clique of $\mathrm{AW}_{p}^{k}$, each node of $S$ contributes to exactly $2 k-s+1$ edges of $\delta(S) \cap \mathrm{AW}_{p}^{k}$, implying that $\left|\delta(S) \cap \mathrm{AW}_{p}^{k}\right|=s(2 k+1-s)$, i.e., (1.7) holds. Take now $S$ of Type 2; then the left side of (1.7) takes value $k(k+1)$ and, since $S_{+}$is an interval of size $s_{+}$with $k+1 \leqslant s_{+} \leqslant p-k$, one computes easily that $\left|\delta(S) \cap \mathrm{AW}_{p}^{k}\right|=k(k+1)$, hence (1.7) holds.

The next proposition, due to Alon [1], is, in fact, the key for validity of CW inequalities.

Proposition 1.12. [1]. Given $p \geqslant 2 k+1$ and a subset $S$ of $[1, p]$ of size $s$, we have:

(i) If $s \leqslant k$, then $\left|\delta(S) \cap \mathrm{AW}_{p}^{k}\right| \geqslant s(2 k+1-s)$.

(ii) If $k \leqslant s \leqslant p-k$, then $\left|\delta(S) \cap \mathrm{AW}_{p}^{k}\right| \geqslant k(k+1)$. 
Claim 1.13. Take $S=S_{+} \cup S_{-}$such that $\delta(S)$ is a root of $\mathrm{CW}_{n}^{k}$, then:

(i) If $s_{+} \leqslant k$, then $s_{-}=0$ and $s_{+}\left(2 k+1-s_{+}\right)=\left|\delta(S) \cap \mathrm{AW}_{p}^{k}\right|$.

(ii) If $k+1 \leqslant s_{+} \leqslant p-k$, then $s_{-}=s_{+}-k$ or $s_{+}-k-1$ and $\left|\delta(S) \cap A W_{p}^{k}\right|=k(k+1)$.

Proof. We state (i). When $s_{+} \leqslant k$, then we have: $s_{+}-s_{-} \leqslant s_{+} \leqslant k$, implying that $\left(s_{+}-s_{-}\right)\left(2 k+1-s_{+}+s_{-}\right) \leqslant s_{+}\left(2 k+1-s_{+}\right) \leqslant\left|\delta(S) \cap \mathrm{AW}_{p}^{k}\right|$; the first inequality follows from the fact that the mapping $x \rightarrow x(2 k+1-x)$ is monotone nondecreasing for $x \leqslant k$ and the latter inequality from Proposition 1.12(i). Using relation (1.7), we deduce that equality, in fact, holds, yielding $s_{-}\left(2 k+1-2 s_{+}+s_{-}\right)=0$ and, thus, $s_{-}=0$ since $2 k+1-2 s_{+}+s_{-} \geqslant 1$. We now state (ii). If $k+1 \leqslant s_{+} \leqslant p-k$, then, from Proposition 1.12(ii), we have that $\left|\delta(S) \cap \mathrm{AW}_{p}^{k}\right| \geqslant k(k+1)$; since $k(k+1)$ is the maximum possible value for $x(2 k+1-x)$ attained at $x=k$ or $k+1$, we deduce from relation (1.7) that equality holds in above inequality and, thus, $s_{+}-s_{-}=k$ or $k+1$.

Claim 1.14. Let $S$ be a subset of $[1, p]$ of size $s$ such that $s \leqslant k$ and $\left|\delta(S) \cap \mathrm{AW}_{p}^{k}\right|=$ $s(2 k+1-s)$, then $S$ induces a clique of $\mathrm{AW}_{p}^{k}$.

Proof. Since every node in the antiweb $\mathrm{AW}_{p}^{k}$ has degree $2 k$, then the number of edges in $\delta(S) \cap \mathrm{AW}_{p}^{k}$ that are incident to each node $x \in S$ is greater or equal to $2 k-(s-1)=2 k+1-s$. By assumption, $\left|\delta(S) \cap \mathrm{AW}_{p}^{k}\right|=s(2 k+1-s)$, implying that there are exactly $2 k+1-s$ edges in $\delta(S) \cap \mathrm{AW}_{p}^{k}$ incident with each node $x \in S$. Therefore, each node $x \in S$ is adjacent in $\mathrm{AW}_{p}^{k}$ to all other nodes of $S$, i.e., $S$ induces a clique of $\mathrm{AW}_{p}^{k}$.

So, in Claim 1.14, we have identified the roots of Type 1 ; we now turn to the case of the roots of Type 2 . We prove by induction on $p \geqslant 2 k+3$ that, for any subset $S$ of size $s$ contained in $[1, p]$, the following assertion hold:

$(\mathrm{H})_{p} \quad$ If $k+1 \leqslant s \leqslant \frac{1}{2} p$ and $\left|\delta(S) \cap \mathrm{AW}_{p}^{k}\right|=k(k+1)$, then $S$ is an interval.

Theorem 1.8 will then follow easily using Claims $1.13,1.14$. Note that, if $(\mathrm{H})_{p}$ holds for $k+1 \leqslant s \leqslant \frac{1}{2} p$ as stated, then it also holds for $k+1 \leqslant s \leqslant p-k-1$ (simply by considering the complement of $S$ ). Observe that the base of induction is $p=2 k+3$, i.e., $q=2$.

Claim 1.15. Assertion $(\mathrm{H})_{p}$ holds for $p=2 k+3$.

Proof. Take $p=2 k+3$; let $C$ denote the cycle with nodeset $[1, p]$ and whose edges are the pairs $(i, i+k+1)$ for $i=1,2, \ldots, p$, where the indices are taken modulo $p$; so $C=(1, k+2, p, k+1, p-1, k, p-2, k-1, \ldots, 2, k+3)$. Obviously, the antiweb $\mathrm{AW}_{2 k+3}^{k}$ is the graph obtained by deleting $C$ from $K_{2 k+3}$. Hence, for $S \subseteq[1, p]$, we 
have: $\left|\delta(S) \cap \mathrm{AW}_{2 k+3}^{k}\right|=s(2 k+3-s)-|\delta(S) \cap C|$. Therefore, if $k+1 \leqslant s \leqslant \frac{1}{2} p$, i.e., $s=k+1$, and $\left|\delta(S) \cap \mathrm{AW}_{2 k+3}^{k}\right|=k(k+1)$, then we deduce that $|\delta(S) \cap C|=$ $2(k+1)=2 s$. This implies easily that no two nodes of $S$ are adjacent on the cycle $C$. If $S$ is not an interval, then $S$ contains two nodes, say 1 and $x$, which are not contained in any interval of size $k+1$, i.e., $x=k+2$ or $k+3$. Since $(1, k+2),(1, k+3)$ are both edges of $C$, we obtain a contradiction.

Take $p \geqslant 2 k+3$ and assume that $(\mathrm{H})_{p}$ holds. We want to prove that $(\mathrm{H})_{p+1}$ still holds; for this, we first establish some connections between the graphs $\mathrm{AW}_{p}^{k}$ and $\mathrm{AW}_{p+1}^{k}$. Considering that $p+1$ is a new node inserted between nodes 1 and $p$ on the cycle $(1,2, \ldots, p)$, one observes easily that the edges belonging to $A W_{p}^{k}$ but not to $\mathrm{AW}_{p+1}^{k}$ are the pairs $(p-k+i, i)$ with $i \in[1, k]$, and the edges belonging to $\mathrm{AW}_{p+1}^{k}$ but not to $\mathrm{AW}_{p}^{k}$ are the pairs $(p+1, i),(p+1, p-k+i)$ with $i \in[1, k]$. This permits us to write

$$
\mathrm{AW}_{p+1}^{k}=\mathrm{AW}_{p}^{k}-\sum_{1 \leqslant i \leqslant k} \Delta_{i}
$$

with $\Delta_{i}=T(i, p+1, p-k+i)$ for $i \in[1, k]$, where $T(a, b, c)$ denotes the triangle on nodes $a, b, c$ with weights +1 on edge $(a, c)$ and -1 on edges $(a, b),(b, c)$ associated with the well-known triangle inequality: $x_{a c}-x_{a b}-x_{b c} \leqslant 0$.

Claim 1.16. If $(\mathrm{H})_{p}$ holds, then $(\mathrm{H})_{p+1}$ holds as well.

Proof. Take $S \subseteq[1, p+1]$ with $k+1 \leqslant s \leqslant \frac{1}{2}(p+1)$ and $\left|\delta(S) \cap \mathrm{AW}_{p+1}^{k}\right|=k(k+1)$. We can assume w.I.o.g. that $p+1 \notin S$. From (1.8), we have that: $\left|\delta(S) \cap A W_{p}^{k}\right|=$ $k(k+1)+\sum_{1 \leqslant i \leqslant k} \Delta_{i}(\delta(S)) \leqslant k(k+1)$, which, together with Proposition 1.12(ii), implies that $\left|\delta(S) \cap \mathrm{AW}_{p}^{k}\right|=k(k+1)$ and, therefore, $\Delta_{i}(\delta(S))=0$ for all $i \in[1, k]$. From $(\mathrm{H})_{p}$, we deduce that $S$ is an interval of $[1, p]$. If the pair $\{1, p\}$ is not contained in $S$, then $S$ is still an interval of $[1, p+1]$. We suppose now that both nodes $1, p$ belong to $S$; then $S=[p-x+1, p] \cup[1, y]$ with $x, y \geqslant 1$ and $x+y \geqslant k+1$. If $x \geqslant k$, then $p-x+1 \leqslant p-k+1$ and, thus, $p-k+1,1 \in S$, implying that $\Delta_{1}(\delta(S))=-2$. If $y \geqslant k$, then $k, p \in S$, implying that $\Delta_{k}(\delta(S))=-2$. Therefore, $x, y \leqslant k-1$. Now, if $x \leqslant y$, then $x \in S$ and $p-x+1 \in S$, yielding $\Delta_{x}(\delta(S))=-2$; else, if $x \geqslant y+1$, then $p-y+1 \in S$, implying that $\Delta_{y}(\delta(S))=-2$. So, we always obtain a contradiction when $\{1, p\} \subseteq S$; this concludes the proof of $(\mathrm{H})_{p+1}$.

\subsubsection{Roots of the general $C W$ inequality}

The general $\mathrm{CW}$ inequality $\mathrm{CW}_{n}^{k}(b)$ comes by $\pi(b)$-collapsing the pure $\mathrm{CW}$ inequality $\mathrm{CW}_{m}^{k}\left(m=\sum_{1 \leqslant i \leqslant n}\left|b_{i}\right|\right)$; hence, using relation (1.5), we obtain that the roots of $\mathrm{CW}_{n}^{k}(b)$ are given by

$$
R\left(\mathrm{CW}_{n}^{k}(b)\right)=\left\{\delta(S): S \subseteq[1, n] \text { and } \delta\left(S^{\pi(b)}\right) \text { is root of } C W_{m}^{k}\right\}
$$


We derive the following property:

Proposition 1.17. For any root $\delta(S)$ of $\mathrm{CW}_{n}^{k}(b)(k \geqslant 1)$, the quantity $b(S)=\sum_{i \in S} b_{i}$ takes its values in $[1, k+1]$.

Proof. If $\delta(S)$ is root of $\mathrm{CW}_{n}^{k}(b)$, then $\delta(T)$ is root of $\mathrm{CW}_{m}^{k}$, where $T=S^{\pi(b)}$. But, $b(S)=t_{+}-t_{-}$, where $t_{+}=|T \cap[1, h]|, t_{-}=|T \cap[h+1, m]|, h=\sum_{1 \leqslant i \leqslant p} b_{i}$. Now, we deduce from Theorem 1.8 that $b(S)$ takes its values in $[1, k+1]$.

For illustration, we describe below the roots of the $\mathrm{CW}$ inequality $\mathrm{CW}_{n}^{k}(2,1, \ldots, 1,-1, \ldots,-1)$ (cf. Example 1.6); the proof is a direct application of (1.9).

Proposition 1.18. The roots of $\mathrm{CW}_{n}^{k}(2,1, \ldots, 1,-1, \ldots,-1)$ (consisting of $p-1$ coefficients $+1, q$ coefficients -1 with $p-q=2 k, n=p+q)$ are the cuts $\delta(S)$ where $S$ is given by:

(1) $S$ is contained in $[1, p]$ and $S$ (resp. $S \cup\{p+1\})$ induces a clique of the antiweb $\mathrm{AW}_{p^{+1}}^{k}$ with $1 \notin S$ (resp. $1 \in S$ ), where $\mathrm{AW}_{p+1}^{k}$ is the antiweb defined on the $p+1$ nodes: $1,2, \ldots, p, p+1$ (taken in that circular order).

(2) $S=S_{+} \cup S_{-}$where $S_{+}$is an interval of $[1, p]$ of size $S_{+}, S_{-}$is a subset of $\left[1^{\prime}, q^{\prime}\right]$ of size s_ with:

- either, $1 \notin S, k+1 \leqslant s_{+} \leqslant p+1-k$ and $s_{-}=s_{+}-k$ or $s_{+}-k-1$,

- or, $1 \in S, k \leqslant s_{+} \leqslant p-k$ and $s_{-}=s_{+}-k+1$ or $s_{+}-k$.

Sometimes, for finding the roots of $\mathrm{CW}_{n}^{k}(b)$, instead of using the idea of collapsing, one can simply look directly at the inequality $\mathrm{CW}_{n}^{k}(b)$ itself, especially when it takes an easy form. This is the case, for instance, for $\mathrm{CW}_{n}^{k}(b)$ with $b_{i} \geqslant k$ for $i \in[1, p]$ (cf. Example 1.5).

Proposition 1.19. The roots of $\mathrm{CW}_{n}^{k}(b)$ with $b_{i} \geqslant k$ for all $i \in[1, p]$ and $b_{i} \leqslant 0$ for $i \in[p+1, n]$ are the cuts $\delta(S)$ for which $S \cap[1, p]$ is an interval and $b(S)=k$ or $k+1$.

Proof. We saw in Example 1.5 that, for $b_{i} \geqslant k$ for $i \in[1, p], \mathrm{AW}_{p}^{k}$ is $\frac{1}{2} k(k+1) C$ where $C$ is the cycle $(1,2, \ldots, p)$. Hence, from $(1.7), \delta(S)$ is root if and only if $b(S)(2 k+1-$ $b(S))$ is equal to $\frac{1}{2} k(k+1)|\delta(S) \cap C|$, that is, $|\delta(S) \cap C|=2$, i.e., $S \cap[1, p]$ is an interval and $b(S)=k$ or $k+1$.

\section{Clique-web facets}

In this paragraph, we give some results on $\mathrm{CW}$ facets. In Section 2.1, we prove that the pure $\mathrm{CW}$ inequalities $\mathrm{CW}_{n}^{k}$ define facets of $C_{n}$ for arbitrary $k$; we prove also that two classes of general $\mathrm{CW}$ inequalities, namely $\mathrm{CW}_{n}^{k}(k, \ldots, k,-1, \ldots,-1)$ and 
$\mathrm{CW}_{n}^{k}(2,1, \ldots, 1,-1, \ldots,-1)$, are classes of general $\mathrm{CW}$ inequalities, namely $\mathrm{CW}_{n}^{k}(k, \ldots, k,-1, \ldots,-1)$ and $\mathrm{CW}_{n}^{k}(2,1, \ldots, 1,-1, \ldots,-1)$, are facet defining (cf. Theorems $2.1,2.2,2.3$ ). In Section 2.2 , we prove that a suitable switching of the pure $\mathrm{CW}$ inequalities also yields facets of the equicut polytope (cf. Theorem 2.5); equicuts are cuts determined by equipartitions of the nodeset. For $n$ odd, the equicut polytope is a facet of the cut polytope and Theorem 2.5 is, in fact, a strengthtening of Theorem 2.1 (cf. Remark 2.6). In Section 2.3, we state a necessary condition for a quasi-linear CW inequality to be facet inducing and we give an upper bound on the norm of $b$ for which $\mathrm{CW}_{n}^{k}(b)$ defines a facet of $C_{n}$. In Section 2.4, we consider the special case of hypermetric inequalities (i.e., $\mathrm{CW}$ inequalities for $k=0$ ); we characterize all facets within the class of hypermetric inequalities of the form $\operatorname{Hyp}_{n}(b)$ with $b$ having $\pm 1, \pm w$ components ( $w$ integer, $w \geqslant 2$ ) (cf. Corollary 2.13) and we give some partial results for the more general class of $\operatorname{Hyp}_{n}(b)$ with the components of $b$ taking values $\pm 1, \pm w, \pm(w+1)$ (cf. Corollary 2.15).

Characterization of all $b$ for which $\mathrm{CW}_{n}^{k}(b)$ is facet inducing seems to be very difficult, even for $k=0$; examples of facets which do not fit in the above classes are those arising from the "uniformisation" procedure in [9] and some sporadical examples checked by computer, e.g., $\mathrm{Cyc}_{8}(2,2,2,1,-1,-1,-1,-1), \mathrm{CW}_{9}^{3}(2,2,2$, $2,1,1,-1,-1,-1)$. The smallest value of $n$ for which there exist facets of $C_{n}$ which are not (permutation or switching) equivalent to some $\mathrm{CW}$ inequalities is $n=7$; known $\mathrm{CW}$ facets for $n=7$ are six hypermetric and three cycle facets (cf. [16, Section 4.3]).

\section{1. $C W$ facets for general $k$}

We use the notation from Section 1: the pure $\mathrm{CW}$ inequality $\mathrm{CW}_{n}^{k}$ is defined on the nodeset $[1, p] \cup\left[1^{\prime}, q^{\prime}\right], n=p+q$ and $p-q=2 k+1$.

Theorem 2.1. The pure $C W$ inequality $\mathrm{CW}_{n}^{k}$, i.e, inequality (1.3), defines a facet of the cut cone $C_{n}$.

Recall that, since zero-lifting preserves facethood, then any pure $\mathrm{CW}$ inequality $\mathrm{CW}_{n}^{k}$ defines a facet of the cut cone $C_{m}$ for any $m \geqslant n$.

In fact, as we explain in Remark 2.6, Theorem 2.1 will follow from Theorem 2.5 which states facethood of pure $\mathrm{CW}$ inequalities for the equicut polytope; so we do not need to prove Theorem 2.1 .

We now give two classes of general $C W$ facets of the cut cone $C_{n}$. We first consider the case of $k$-uniform linear $\mathrm{CW}$ inequality $\mathrm{CW}_{n}^{k}(k, \ldots, k,-1, \ldots,-1)$ consisting of $p$ coefficients $k, q$ coefficients -1 with $q \geqslant 2, n=p+q, p k-q=2 k+1$ and $k \geqslant 1$. In this case, the collapsed antiweb $\mathrm{AW}_{p}^{k}(k, \ldots, k)$ is simply the cycle $(1,2, \ldots, p)$ with weight $\frac{1}{2} k(k+1)$ on its edges (Example 1.5) and, as corollary of Proposition 1.19 , the roots are the cut vectors $\delta(S)$ for which $S=S_{+} \cup S_{-}$where $S_{+}$is an interval of $[1, p]$ of size $s_{+}, S_{-}$is a subset of $\left[1^{\prime}, q^{\prime}\right]$ of size $s_{-}$with $k s_{+}-s_{-}=k$ or $k+1$. 
Theorem 2.2. The $C W$ inequality $\mathrm{CW}_{n}^{k}(k, \ldots, k,-1, \ldots,-1)$ is facet inducing in $C_{n}$ for all $k \geqslant 1, p \geqslant 5$.

We consider now the case $\mathrm{CW}_{n}^{k}(2,1, \ldots, 1,-1, \ldots,-1)$ where there are $p-1$ coefficients $+1, q$ coefficients -1 with $q \geqslant 2, p-q=2 k, k \geqslant 1, n=p+q$. The collapsed antiweb $\mathrm{AW}_{p}^{k}(2,1, \ldots, 1)$ was described in Example 1.6 and Proposition 1.18 gives the description of the roots.

Theorem 2.3. With the above notation, the $C W$ inequality $\mathrm{CW}_{n}^{k}(2,1, \ldots, 1,-1, \ldots$, $-1)$ is facet inducing for all $k \geqslant 1$ and $p \geqslant 2 k+3$.

The proofs of Theorem 2.2 and 2.3 are given in Sections 3.2 and 3.3.

Using some lifting techniques developed in $[15,17]$, the following class of $\mathrm{CW}$ facets is given in [17]: $\mathrm{CW}_{n}^{r-s}\left(b_{1}, \ldots, b_{p},-1, \ldots,-1,-2, \ldots,-2\right)$ with $q-s$ coefficients equal to $-1, s$ coefficients equal to $-2, b_{1}, \ldots, b_{p} \geqslant r \geqslant s \geqslant 0, p \geqslant 5$, $q=b_{1}+\cdots+b_{p}+s-2 r-1$ and $n=p+q$. Also, it is proved in [17] that, if $b_{1} \geqslant \cdots \geqslant$ $b_{p} \geqslant k$, the CW inequality $\mathrm{CW}_{n}^{k}\left(b_{1}, \ldots, b_{p},-1, \ldots,-1\right)$ (with $q=n-p$ coefficients -1 and $\left.\sum_{1 \leqslant i \leqslant p} b_{i}-q=2 k+1\right)$ is facet defining if and only if $p \geqslant 5$, or $(p=4$ and $b_{1}, b_{2} \geqslant k+1$, up to cyclic shift on $\left.[1,4]\right)$, or $\left(p=3, b_{1} \geqslant k+2, b_{2}, b_{3} \geqslant k+1\right.$, up to cyclic shift on $[1,3])$.

\subsection{CW facets for the equicut polytope}

In this section, we show that a suitable switching of the pure $\mathrm{CW}$ inequalities also yields facets of the equicut polytope. Equicuts correspond to partitions of the nodes into (almost) equal parts and they have applications in various domains, in particular, in statistical physics (for the determination of ground states of spin glasses with zero magnetization ) (see [5]).

Our study of $\mathrm{CW}$ facets for the equicut polytope is motivated, first, by the intrinsic interest of the equicut polytope in view of its applications, and second, by the fact that, if we can prove that some switching of the $\mathrm{CW}$ inequalities defines a facet of the equicut polytope, then, necessarily, it also defines a facet of the cut polytope.

A cut $\delta(S)$ of $K_{n}$ is called an equicut if $|S|=\left\lfloor\frac{1}{2} n\right\rfloor$ or $\left\lceil\frac{1}{2} n\right\rceil$; a cut which is not an equicut is called an inequicut. We denote by $\mathrm{EP}_{n}$ the equicut polytope, i.e., the convex hull of all equicuts of $C_{n}$ and we denote by $\mathrm{IC}_{n}$ the inequicut cone, i.e., the cone generated by all inequicuts. The inequicut cone and its relatives are considered in [12]; in particular, $\mathrm{IC}_{n}$ is full dimensional for $n \geqslant 5$ and it is shown in [12] that any facet of $C_{n}$ (hence any CW facet) defines, in fact, a facet of $\mathrm{IC}_{m}$ for all $m \geqslant 2 n$. The equicut polytope was studied in [8]; for $n$ odd, $\mathrm{EP}_{n}$ has dimension $\frac{1}{2} n(n-1)-1$ and, in fact, is the facet of the cut polytope $P_{n}$ induced by the valid inequality $\sum_{1 \leqslant i<j \leqslant n} x_{i j} \leqslant \frac{1}{4}\left(n^{2}-1\right)[8]$, the inequality $\mathrm{CW}_{n}^{k}$ itself is valid for $\mathrm{EP}_{n}$, but is not facet defining. For this, observe that, with the notation of Theorem 1.8 and Remark 1.9, no root of Type 1 of $\mathrm{CW}_{n}^{k}$ is an equicut and the only roots of Type $2^{\prime}$ which are equicuts are the cuts $\delta(S)$ with $S=S_{+} \cup S_{-}$and $s_{+}=s_{-}+k, s_{-}=\frac{1}{2} q$ or $\frac{1}{2}(q+1)$. 
Therefore, the face of $\mathrm{EP}_{n}$ induced by the inequality $\mathrm{CW}_{n}^{k}$ is contained in the face of $\mathrm{EP}_{n}$ induced by the valid inequality $\sum_{p+1 \leqslant i<j \leqslant n} x_{i j} \leqslant\left\lfloor\frac{1}{2} q\right\rfloor\left\lceil\frac{1}{2} q\right\rceil$ and, hence, is not a facet of $C_{n}$. However, the inequality $\mathrm{CW}_{n}^{k}$ defines a facet of $\mathrm{EP}_{m}$ for all $m$ odd, $m \geqslant 2 n+1$ ([12]). On the other hand, some suitable switching of $\mathrm{CW}_{n}^{k}$ will give a facet of $\mathbf{E P}_{m}$ for all $m$ odd, $m \geqslant n+2$.

We consider now the following inequality $(2.1)$, also denoted by $\left(C W_{n}^{k}\right)^{\left[1^{1}, q^{\prime}\right]}$, obtained by switching the inequality $(1.3)$ by the cut $\delta\left(\left[1^{\prime}, q^{\prime}\right]\right)$ :

$$
\left(\mathrm{CW}_{n}^{k}\right)^{\left[1^{\prime}, q^{\prime}\right]} \cdot x=\sum_{(i, j) \in K_{n}-\mathrm{AW} W_{p}^{k}} x_{i j} \leqslant p q .
$$

Remark 2.4. As a corollary of Theorem 1.8 , we can describe the roots of $\left(\mathrm{CW}_{n}^{k}\right)^{\left[1^{\prime}, q^{\prime}\right]}$; they are exactly the cuts $\delta\left(S \triangle\left[1^{\prime}, q^{\prime}\right]\right)$ for which $\delta(S)$ is root of $\mathrm{CW}_{n}^{k}$, hence they are the cuts $\delta(S)$ for which $S$ is of one of the following two types:

Type 1: $S=S_{+} \cup\left[1^{\prime}, q^{\prime}\right]$ where $S_{+} \subseteq[1, p]$ induces a clique of $\mathrm{AW}_{p}^{k}$.

Type 2: $S=S_{+} \cup S_{-}$where $S_{+}$is an interval of size $s_{+}$of $[1, p], k+1 \leqslant s_{+} \leqslant p-k$, $S_{-}$is a subset of $\left[1^{\prime}, q^{\prime}\right]$ of size $s_{-}$and $s_{-}=q+k-s_{+}$or $q+k+1-s_{+}$.

Since $n=2(q+k)+1$, we observe that, for all $S$ of Type $2, \delta(S)$ is, in fact, an equicut, while the sets $S$ of type 1 for which $\delta(S)$ is an equicut are those having $s_{+}=k$ or $k+1$.

Theorem 2.5. The inequality (2.1) defines a facet of the equicut polytope $\mathrm{EP}_{m}$ for all $m$ odd, $m \geqslant n+2$ and, moreover, for $m=n$ when $k=1$.

This result extends Theorems 6.2, 6.3 and 6.4 from [8] which correspond to the extremal cases $k=1$ (i.e., up to switching, pure cycle inequality) and $k=\frac{1}{2}(n-5)$ or, equivalently, $p=2 k+3$ (i.e., the bicycle odd wheel inequality). The bound $m \geqslant n+2$ in Theorem 2.5 can probably be improved to $m \geqslant n$ for some parameters $n, k$. The proof of Theorem 2.5 is given in Section 3.1.

Remark 2.6. Theorem 2.1 follows from Theorem 2.5, as we now indicate (see [12] for more details on the connections between facets of the cut and equicut polytopes).

(i) Since the inequality (2.1) is a switching of the inequality (1.3), if we can prove that (2.1) defines a facet of the cut polytope $P_{n}$, then this implies that (1.3) defines a facet of the cut cone $C_{n}$.

(ii) If we can prove that (2.1) defines a facet of the cut polytope $P_{n+2}$, then this implies that (2.1) defines a facet of $P_{n}$ (recall the property of zero-lifting mentioned in the Introduction).

(iii) From Theorem 2.5, (2.1) defines a facet of the equicut polytope $\mathrm{EP}_{n+2}$; hence, one can find a set of $\left(\begin{array}{c}n+2 \\ 2\end{array}\right)-1$ affinely independent equicut vectors which are roots of (2.1). If one adjoins to this set one more root which is not an equicut, say $\delta\left(\left[1^{\prime}, q^{\prime}\right]\right)$, then it is clear that this set of $\left(\begin{array}{c}n+2 \\ 2\end{array}\right)$ roots is affinely independent. Therefore, (2.1) defines a facet of $P_{n+2}$. 


\subsection{A necessary condition for a $C W$ inequality to be facet inducing}

Facets are, by definition, maximal faces of the cut cone which means that they are not contained in any valid inequality. The $\mathrm{CW}$ inequality $\mathrm{CW}_{n}^{k}(b)$ is called quasilinear if all negative coefficients $b_{i}$ of $b$ except at most one are equal to -1 . We derive below a necessary condition for quasilinear $\mathrm{CW}$ inequalities to be facet inducing; it is based on the trivial fact that a $\mathrm{CW}$ facet cannot be contained in any triangle facet.

Consider a quasilinear $\mathrm{CW}$ inequality $\mathrm{CW}_{n}^{k}(b)$, i.e., $b=\left(b_{1}, \ldots, b_{p},-1, \ldots\right.$, $\left.-1, b_{n}\right)$ with $b_{1}, \ldots, b_{p}>0, b_{n}<0, \sum b_{i}=2 k+1$ and consisting of $q-1$ coefficients -1 . We denote by $b_{i}, b_{j}$ the two largest coefficients among $b_{1}, \ldots, b_{p}$.

Proposition 2.7. If a quasilinear $C W$ inequality $\mathrm{CW}_{n}^{k}(b)$ is facet inducing, then the following condition holds:

$$
b_{i}+b_{j} \leqslant q+k \text {. }
$$

Proof. Let us suppose that $\mathrm{CW}_{n}^{k}(b)$ is facet inducing and that $b_{i}+b_{j} \geqslant q+1+k$. If $\delta(S)$ is root of $\mathrm{CW}_{n}^{k}(b)$, then we can suppose w.l.o.g. that $n \notin S$ and we have that $b(S) \in[1, k+1]$ when $k \geqslant 1$ (from Proposition 1.17) and $b(S)=0,1$ when $k=0$. If both nodes $i, j$ belong to $S$, then we have that: $b(S) \geqslant b_{i}+b_{j}-(q-1) \geqslant 2+k$, yielding a contradiction. Hence, $\{i, j\} \nsubseteq S$ for all roots $\delta(S)$ of $\mathrm{CW}_{n}^{k}(b)$. This implies that all roots of $\mathrm{CW}_{n}^{k}(b)$ are, in fact, roots of the triangle inequality: $x_{i j}-x_{i n}-x_{j n} \leqslant 0$, contradicting the assumption that $\mathrm{CW}_{n}^{k}(b)$ is facet inducing.

Observe that, what we proved is, that if (2.2) does not hold, then the face defined by $\mathrm{CW}_{n}^{k}(b)$ is contained in some triangle facet.

Remark 2.8. For $k=0$, condition (2.2) is $b_{i}+b_{j} \leqslant q=n-p$. Theorem 3.12 [16] gives a complete characterization of quasilinear hypermetric facets and can be rephrased as follows. In the uniform case, i.e., $b_{1}=\cdots=b_{p}$, the necessary and sufficient condition for facethood is $b_{i}+b_{j} \leqslant q-1$ and, otherwise (in non-uniform case), the necessary and sufficient condition is exactly (2.2) $b_{i}+b_{j} \leqslant q$. In fact, as a biproduct of the proof of Proposition 2.7, one obtains that any non-uniform quasilinear hypermetric face is, either a facet, or is contained in a triangle facet. We will see in the next section other classes of hypermetric inequalities for which the same phenomenon occurs: they are either facets, or contained in some pure hypermetric facet.

The bound (2.2) is sharp. For instance, the facets $\mathrm{CW}_{n}^{1}(n-5,2,1,1,-1, \ldots,-1)$ (cf. [16, Theorem 3.27]), $\mathrm{CW}_{n}^{1}\left(b_{1}, b_{2}, 2,-1, \ldots,-1\right)$ with $b_{1}+b_{2}=n-2$ (cf. [16, Theorem 3.26]) give equality in (2.2); the non-uniform quasilinear hypermetric facet $\operatorname{Hyp}_{n}\left(b_{1}, \ldots, b_{p},-1, \ldots,-1, b_{n}\right)$ gives equality if and only if $b_{n}=-\left(b_{3}+\cdots+b_{p}\right)$.

We conclude this section by giving bounds on $g_{k}(n)=\max \left(\left\{\|b\|: \mathrm{CW}_{n}^{k}(b)\right.\right.$ is facet inducing for $\left.C_{n}\right\}$ ), setting $\|b\|=\sum_{1 \leqslant i \leqslant n}\left|b_{i}\right|$. First, the CW facet from Theorem 2.2 
has $\|b\|=2 n k /(k+1)+(2 k+1)(k-1) /(k+1)$; hence, this gives a lower bound on $g_{k}(n)$ for $k \geqslant 1$ which is linear in $n$. Let $\beta_{n}$ denote the maximum value of an $n \times n$ determinant with binary entries. It was proved in [4] that $g_{0}(n) \leqslant n \beta_{n-2}$ and, in [16], that $g_{1}(n) \leqslant 3+4(n-1)^{2} \beta_{n-2}$. One can extend easily this result for any $k \geqslant 1$.

Proposition 2.9. $g_{k}(n) \leqslant 2 k+1+2(n-1)^{2}(k+1) \beta_{n-2}$ for $k \geqslant 1$.

The bound from Proposition 2.9 is exponential in $n$; it would be interesting to find a polynomial upper bound. The problem of finding good estimates of $g_{k}(n)$ is also related to the problem of determining whether the cone $\mathrm{CW}(n, k)$ which is defined as the solution set of the $\mathrm{CW}$ inequalities $\mathrm{CW}_{n}^{k}(b)$ for all possible $b$ whose sum is $2 k+1$ is polyhedral. Polyhedrality of the cone $\mathrm{CW}(n, k)$ means that $\|b\|$ is bounded for all $b$ such that $\mathrm{CW}_{n}^{k}(b)$ is facet inducing for the cone $\mathrm{CW}(n, k)$. For $k=0$, the cone $\mathrm{CW}(n, 0)$ is indeed polyhedral [14].

\subsection{New hypermetric facets}

Let $w, \alpha, \alpha^{\prime}, \beta, \beta^{\prime}$ be integers satisfying the condition

$$
\left(\alpha-\alpha^{\prime}\right) w+\beta-\beta^{\prime}=1 .
$$

In this section, we characterize hypermetric facets of the form $\operatorname{Hyp}_{n}(w, \ldots, w$, $-w, \ldots,-w, 1, \ldots, 1,-1, \ldots,-1)$, consisting of $\alpha$ coefficients $+w, \alpha^{\prime}$ coefficients $-w, \beta$ coefficients +1 and $\beta^{\prime}$ coefficients -1 with $\alpha, \alpha^{\prime}, \beta, \beta^{\prime}$ satisfying condition (2.3) and $n=\alpha+\alpha^{\prime}+\beta+\beta^{\prime}$; for short, we denote the above hypermetric inequality by $\operatorname{Hyp}_{n}\left((w)_{\alpha},(-w)_{\alpha^{\prime}},(1)_{\beta},(-1)_{\beta^{\prime}}\right)$. When $w=1$ or $\alpha^{\prime}=1$, we obtain a quasilinear hypermetric inequality; in this case, complete characterization is known (recall Remark 2.8). So we can suppose that $w \geqslant 2$ in the following, which, from (2.3), implies that $\min \left(\beta, \beta^{\prime}\right) \geqslant 1$. Examples of hypermetric facets which are not quasilinear include $\operatorname{Hyp}_{11}(2,2,2,-2,-2,-2,1,1,1,-1,-1)$, $\operatorname{Hyp}_{15}(3,3,-3,-3,-3,1,1,1$, $1,1,1,1,-1,-1,-1)$ and $\operatorname{Hyp}_{19}(4,4,-4,-4,3,3,-3,-3,-3,1,1,1,1,1,1,1$, $-1,-1,-1)$; they are obtained through the following Corollaries 2.13, 2.15.

Theorem 2.10. Assume that $\operatorname{Hyp}_{n}\left((w)_{\alpha},(-w)_{\alpha^{\prime}},(1)_{\beta},(-1)_{\beta^{\prime}}\right)$ is facet inducing with $\alpha \geqslant \alpha^{\prime} \geqslant 1$. If one of the following two conditions holds:

(i) $\alpha \geqslant \alpha^{\prime}+1$,

(ii) $\alpha=\alpha^{\prime}$ and $\beta \geqslant w$,

then, $\operatorname{Hyp}_{n+2}\left((w)_{\alpha+1},(-w)_{\alpha^{\prime}+1},(1)_{\beta},(-1)_{\beta^{\prime}}\right)$ is facet inducing.

Proof. The proof is based on the lifting technique introduced in [16], we use all definitions and results on lifting from Section 2.2 in [16]. We set $b=\left((w)_{\alpha},(-w)_{\alpha}\right.$, $\left.(1)_{\beta},(-1)_{\beta^{\prime}}\right)$. Let $A, A^{\prime}, B, B^{\prime}$ denote, respectively, the sets of positions $i \in[1, n]$ for which $b_{i}=w,-w, 1,-1$; hence their respective cardinalities are $\alpha, \alpha^{\prime}, \beta, \beta^{\prime}$. Since $\operatorname{Hyp}_{n}(b)$ is facet inducing, then $\operatorname{Hyp}_{n+1}(0, b)$ is also facet inducing. In order to prove that $\operatorname{Hyp}_{n+2}(-w, b, w)$ is facet defining, using Proposition 2.7 of [16], it 
suffices to show that we can find $n+1$ roots $\delta(S)$ of $\operatorname{Hyp}_{n+2}(-w, b, w)$ with $1 \notin S$, $n+2 \in S(1, n+2$ being the positions corresponding respectively to the new coefficients $-w, w$ in the $(n+2)$-vector $(-w, b, w))$ whose incidence matrix is nonsingular.

From the assumptions, we have that $\alpha^{\prime}, \beta \geqslant 1$; we consider fixed nodes $i^{\prime} \in A^{\prime}$, $j \in B$. Observe that $\beta^{\prime} \geqslant w-1$ always holds; indeed, if $\alpha-\alpha^{\prime} \geqslant 1$, then, from (2.3), $\beta^{\prime}=\left(\alpha-\alpha^{\prime}\right) w+\beta-1 \geqslant w-1$ and, if $\alpha=\alpha^{\prime}$, then $\beta^{\prime}=\beta-1 \geqslant w-1$ by assumption (ii). Hence, we can consider a subset $X$ of $B^{\prime}$ of size $w-1$. We choose the following $n+1$ roots $\delta(S)$ :

$$
\begin{aligned}
& S=\{i, n+2\} \text { for } i \in A^{\prime}, \\
& S=\left\{i^{\prime}, i, n+2\right\} \text { for } i \in B, \\
& S=\left\{i^{\prime}, j, i, n+2\right\} \quad \text { for } i \in B^{\prime}, \\
& S=\left\{i^{\prime}, i, n+2\right\} \cup X \text { for } i \in A, \\
& S=X .
\end{aligned}
$$

One verifies easily that their incidence matrix has rank $n+1$.

Theorem 2.11. Given $\alpha, \beta \geqslant 1, \operatorname{Hyp}_{n}\left((w)_{\alpha},(-w)_{\alpha},(1)_{\beta},(-1)_{\beta-1}\right)$ is facet inducing if and only if $\beta \geqslant w+1$.

Proof. Suppose first that $\beta \geqslant w+1$. Using the known characterization of quasilinear hypermetric facets stated in Remark 2.8, we deduce that $\operatorname{Hyp}\left(w,-w,(1)_{\beta},(-1)_{\beta-1}\right)$ is facet inducing. Starting with this facet, we apply iteratively $(\alpha-1$ times) Theorem 2.10 until obtaining the desired facet $\operatorname{Hyp}_{n}\left((w)_{\alpha},(-w)_{\alpha},(1)_{\beta},(-1)_{\beta-1}\right)$. Conversely, we suppose that $\beta \leqslant w$. Set $v=\operatorname{Hyp}_{n}\left((w)_{\alpha},(-w)_{\alpha},(1)_{\beta},(-1)_{\beta-1}\right)$. Let $\delta(S)$ be a root of $v$; let $s, s^{\prime}, t, t^{\prime}$ denote, respectively, the number of nodes $i \in S$ with $b_{i}=w$, $-w, 1,-1$; then, $s, s^{\prime} \leqslant \alpha, t \leqslant \beta, t^{\prime} \leqslant \beta-1$ and they satisfy: $w\left(s-s^{\prime}\right)+t-t^{\prime}=0$ or 1 . Let $i$ be a node with $b_{i}=1$; we can suppose that $i \notin S$ for all roots $\delta(S)$ of $v$, i.e., $t \leqslant \beta-1$. If $s-s^{\prime} \geqslant 2$, then, $w\left(s-s^{\prime}\right)+t-t^{\prime} \geqslant 2 w-\beta+1 \geqslant w+1>1$; If $s-s^{\prime} \leqslant-2$, then, $w\left(s-s^{\prime}\right)+t-t^{\prime} \leqslant-2 w+\beta \leqslant-w<0$ and, if $s-s^{\prime}=-1$, then, $w\left(s-s^{\prime}\right)+t-t^{\prime}=$ $t-t^{\prime}-w$ which is equal to 0 or 1 if and only if $\beta=w$ and $t=w, t^{\prime}=0$. Therefore, the cases $\left|s-s^{\prime}\right| \geqslant 2, s-s^{\prime}=-1$ are excluded and, thus, $s-s^{\prime}$ is equal to 0 or 1 . This means exactly that every root of $v$ is, in fact, a root of $\operatorname{Hyp}\left((1)_{\alpha},(-1)_{\alpha}, 1,(0)_{2 \beta-2}\right)$ (where the last " 1 " is in position $i$ ); in other words, the face induced by $v$ is contained in some pure hypermetric facet and thus is not facet inducing.

Theorem 2.12. Given $\alpha \geqslant 1, \beta \geqslant 0, \operatorname{Hyp}_{n}\left((w)_{\alpha+1},(-w)_{\alpha},(1)_{\beta},(-1)_{\beta+w-1}\right)$ is facet inducing if and only if $\beta \geqslant w$.

Proof. We suppose first that $\beta \geqslant w$. If $\alpha=1$, the given hypermetric inequality is quasilinear and facet inducing using Remark 2.8 . Then, we iteratively apply Theorem 
2.10 until obtaining the desired facet. We suppose now that $\beta \leqslant w-1$. Set $v=$ $\operatorname{Hyp}_{n}\left((w)_{\alpha+1},(-w)_{\alpha},(1)_{\beta},(-1)_{\beta+w-1}\right)$. Using the same notation as in the proof of Theorem 2.11 for $s, s^{\prime}, t, t^{\prime}$, then $\delta(S)$ is root if and only if $w\left(s-s^{\prime}\right)+t-t^{\prime}=0$ or 1. Similarly, if $s-s^{\prime} \geqslant 2$, then, $w\left(s-s^{\prime}\right)+t-t^{\prime} \geqslant w-\beta+1 \geqslant 2$ and, if $s-s^{\prime} \leqslant-1$, then, $w\left(s-s^{\prime}\right)+t-t^{\prime} \leqslant-w+\beta \leqslant-1$. Therefore, we must have: $s-s^{\prime}=0$ or 1 ; hence, the face induced by $v$ is contained in the pure hypermetric facet $\operatorname{Hyp}_{n}\left((1)_{\alpha+1},(-1)_{\alpha}\right.$, $\left.(0)_{2 \beta+w-1}\right)$ and thus $v$ is not facet inducing.

Corollary 2.13. Given $\alpha, \alpha^{\prime}, \beta, \beta^{\prime}$ satisfying (2.3) and $\min \left(\alpha, \alpha^{\prime}\right) \geqslant 1$ and setting $v=\operatorname{Hyp}_{n}\left((w)_{\alpha},\left(-w^{\prime}\right)_{\alpha^{\prime}},(1)_{\beta},(-1)_{\beta}\right)$, the following assertions hold:

(i) If $\alpha=\alpha^{\prime}$ (i.e., $\beta^{\prime}=\beta-1$ ), then $v$ is facet defining if and only if $\min \left(\beta, \beta^{\prime}\right) \geqslant w$.

(ii) If $\left|\alpha-\alpha^{\prime}\right|$ is a nonzero even number, then $v$ is facet defining if and only if $\min \left(\beta, \beta^{\prime}\right) \geqslant 1$ or $\left(\min \left(\beta, \beta^{\prime}\right)=0\right.$ and $\left.\left|\alpha-\alpha^{\prime}\right| \geqslant 4\right)$.

(iii) If $\left|\alpha-\alpha^{\prime}\right|$ is an odd number, then $v$ is facet defining if and only if $\left|\alpha-\alpha^{\prime}\right| \geqslant 3$ or $\left(\left|\alpha-\alpha^{\prime}\right|=1\right.$ and $\left.\min \left(\beta, \beta^{\prime}\right) \geqslant w\right)$.

Proof. Assertion (i) is just Theorem 2.11. We suppose that $\alpha \neq \alpha^{\prime}$. Given $\gamma \geqslant 0$, one observes easily that $\operatorname{Hyp}\left((w)_{\alpha},(-w)_{\alpha+\gamma},(1)_{\beta^{\prime}+\gamma w+1},(-1)_{\beta^{\prime}}\right)$ and $\operatorname{Hyp}\left((w)_{\alpha+\gamma}\right.$, $\left.(-w)_{\alpha},(1)_{\beta^{\prime}},(-1)_{\beta^{\prime}+\gamma w+1}\right)$ are switching equivalent (hint: for obtaining the second one, switch the first one by the root $\delta(S)$ where $S$ consists of $\gamma$ nodes $i$ with $b_{i}=-w$ and $\gamma w+1$ nodes $i$ with $b_{i}=1$ ). Therefore, in order to characterize facets in cases (ii), (iii), we can suppose that $\alpha>\alpha^{\prime}$, i.e., $\alpha=\alpha^{\prime}+\gamma$ with $\gamma>0$ and $\beta \leqslant \beta^{\prime}$. We distinguish two cases:

Case A. $\gamma=2 \eta$ is even, $\eta>0$. Observe then that $\operatorname{Hyp}\left((w)_{\alpha^{\prime}+2 \eta},(-w)_{\alpha^{\prime}},(1)_{\beta}\right.$, $\left.(-1)_{2 w \eta+\beta-1}\right)$ and $\operatorname{Hyp}\left((w)_{\alpha^{\prime}+\eta},(-w)_{\alpha^{\prime}+\eta},(1)_{\beta+w^{\prime}},(-1)_{\beta+w^{\prime} \eta-1}\right)$ are switching equivalent. From Theorem 2.11 , the latter is facet inducing if and only if $\beta+\eta w \geqslant$ $w+1$, i.e., either $\eta \geqslant 2$, or $\eta=1$ and $\beta \geqslant 1$, thus stating (ii).

Case B. $\gamma=2 \eta+1$ is odd, $\eta \geqslant 0$. Then, $\operatorname{Hyp}\left((w)_{\alpha^{\prime}+2 \eta+1}, \quad(-w)_{\alpha^{\prime}}, \quad(1)_{\beta}\right.$, $\left.(-1)_{\beta+(2 \eta+1) w-1}\right)$ and $\operatorname{Hyp}\left((w)_{\alpha^{\prime}+\eta+1},(-w)_{\alpha^{\prime}+\eta},(1)_{\beta+w \eta},(-1)_{\beta+w \eta+w-1}\right)$ are switching equivalent. From Theorem 2.12, the latter is facet inducing if and only if $\beta+\eta w \geqslant w$, i.e., either $\eta \geqslant 1$, or $\eta=0$ and $\beta \geqslant w$, thus stating (iii).

We completely characterized hypermetric facets $\operatorname{Hyp}_{n}(b)$ with $b$ admitting four possible values: $w,-w, 1,-1$. Starting with these new facets, one can try to further lift them and, in particular, study hypermetric facets with six possible components of $b: u,-u, w,-w, 1,-1$. We give some partial results in this direction for the case $u=w+1$.

Theorem 2.14. Assume that $\mathrm{Hyp}_{n}\left((w+1)_{\gamma},(-w-1)_{\gamma},(w)_{\alpha},(-w)_{\alpha^{\prime}},(1)_{\beta},(-1)_{\beta^{\prime}}\right)$ is facet inducing with $\min \left(\alpha, \alpha^{\prime}\right) \geqslant 1$. If $\beta \geqslant w$ holds in the case $\alpha=\alpha^{\prime}$ and $\gamma \geqslant 1$, then $\operatorname{Hyp}_{n+2}\left((w+1)_{\gamma+1},(-w-1)_{\gamma+1},(w)_{\alpha},(-w)_{\alpha^{\prime}},(1)_{\beta},(-1)_{\beta^{\prime}}\right)$ is facet defining.

Proof. As for the proof of Theorem 2.10, we use the lifting technique, i.e., if $1, n+2$ are the positions corresponding respectively to the new coefficients $-w-1$ and $w+1$, 
we show that we can find $n+1$ roots of $\operatorname{Hyp}_{n+2}\left((w+1)_{\gamma_{+1}},(-w-1)_{\gamma_{+1}},(w)_{\alpha},(-w)_{\alpha^{\prime}}\right.$, $\left.\left.(1)_{\beta},(-1)_{\beta^{\prime}}\right)\right)$ with $1 \notin S, n+2 \in S$ and whose incidence matrix has rank $n+1$. Up to switching, we can suppose that $\alpha \geqslant \alpha^{\prime}$. As in the proof of Theorem 2.10, we define the sets $A, A^{\prime}, B, B^{\prime}$. We introduce $C, C^{\prime}$ as the sets of positions $i$ with $b_{i}=w+1$, $-w-1$, respectively.

Case A. $\gamma=0$. From the assumptions and Corollary 2.13, we deduce that $\beta^{\prime} \geqslant w$. Let $X$ be a subset of $B^{\prime}$ of size $w$ and $i^{\prime} \in A^{\prime}, j \in X$. We choose the following $n+1$ roots $\delta(S)$ :

$$
\begin{aligned}
& S=\{i, n+2\} \quad \text { for } i \in A^{\prime}, \\
& S=\left\{i^{\prime}, i, n+2\right\} \quad \text { for } i \in A, \\
& S=\left\{i^{\prime}, i, n+2\right\} \quad \text { for } i \in B^{\prime}, \\
& S=\left\{i^{\prime}, j, i, n+2\right\} \quad \text { for } i \in B, \\
& S=X .
\end{aligned}
$$

One checks easily that their incidence matrix is nonsingular.

Case B. $\gamma \geqslant 1$. Take $i^{\prime} \in A^{\prime}, h \in C, h^{\prime} \in C^{\prime}$. We choose the following $n+1$ roots $\delta(S)$ :

$$
\begin{aligned}
& S=\{i, n+2\} \quad \text { for } i \in C^{\prime}, \\
& S=\{i, n+2\} \text { for } i \in A^{\prime}, \\
& S=\left\{i, i^{\prime}, h^{\prime}, n+2\right\} \quad \text { for } i \in C, \\
& S=\left\{i, i^{\prime}, h^{\prime}, n+2\right\} \quad \text { for } i \in A, \\
& S=\left\{i, i^{\prime}, n+2\right\} \quad \text { for } i \in B^{\prime}, \\
& S=\left\{i, h^{\prime}, n+2\right\} \text { for } i \in B,
\end{aligned}
$$

and

$$
S=Y \cup\left\{i^{\prime}, h^{\prime}, n+2\right\}
$$

where $Y$ is a subset of $B$ of size $w$ when $\alpha=\alpha^{\prime}$ or

$$
S=A \cup A^{\prime} \cup B \cup B^{\prime} \cup C-\{h\} \cup C^{\prime}
$$

when $\alpha>\alpha^{\prime}$.

One checks easily that their incidence matrix is nonsingular.

Corollary 2.15. If $\operatorname{Hyp}\left((w)_{\alpha},(-w)_{\alpha^{\prime}},(1)_{\beta},(-1)_{\beta^{\prime}}\right)$ is facet inducing, then $\operatorname{Hyp}((w+$ $\left.1)_{\gamma},(-w-1)_{\gamma},\left(w_{\alpha}\right),(-w)_{\alpha^{\prime}},(1)_{\beta},(-1)_{\beta^{\prime}}\right)$ too is facet inducing, for any $y \geqslant 0$.

Proof. The proof follows directly from Corollary 2.13 and iterated applications of Theorem 2.14.

\section{Proofs for clique-web facets}

In this section, we give the proofs of Theorems $2.5,2.2$ and 2.3 concerning facethood of the pure $\mathrm{CW}$ inequality $\mathrm{CW}_{n}^{k}$ for the equicut polytope and the two general $\mathrm{CW}$ 
inequalities $\mathrm{CW}_{n}^{k}(k, \ldots, k,-1, \ldots,-1)$ and $\mathrm{CW}_{n}^{k}(2,1, \ldots, 1,-1, \ldots,-1)$ for the cut cone. The proofs are based on the polyhedral method. Namely, in order to prove that a given valid inequality $v \cdot x \leqslant 0$ is facet inducing for $C_{n}$, we show that, if $a \cdot x \leqslant 0$ is another valid inequality for $C_{n}$ such that $\left\{x \in C_{n}: v \cdot x=0\right\} \subseteq$ $\left\{x \in C_{n}: a \cdot x=0\right\}$, then $a=\alpha v$ for some scalar $\alpha$ ( $\alpha$ is then necessarily positive). We recall in Section 3.1 the characterization for facets of $E P_{n}$. We state two lemmas that we will use thoroughly in the proofs; they follow respectively from Lemmas 2.5 in $[7,6]$.

Lemma 3.1. Let a be a vector of length $\frac{1}{2} n(n-1)$ indexed by $K_{n}$. Let $i, j$ be distinct elements of $[1, n]$ and $S$ be a subset (possibly empty) of $[1, n]-\{i, j\}$ such that the cuts $\delta(S), \delta(S \cup\{i\}), \delta(S \cup\{j\})$ and $\delta(S \cup\{i, j\})$ satisfy equality: $a \cdot x=0$. Then, $a_{i j}=0$ holds.

Lemma 3.2. Let a be a vector of length $\frac{1}{2} n(n-1)$ indexed by $K_{n}$. Let $I, J, H$ and $S$ be disjoint subsets of $[1, n]$ such that the cuts $\delta(S \cup J), \delta(S \cup H), \delta(S \cup I \cup J)$ and $\delta(S \cup I \cup H)$ satisfy equality $a \cdot x=0$. Then, $\sum_{i \in I, j \in J} a_{i j}=\sum_{i \in I, h \in H} a_{i h}$ holds. In particular, if $I=\{i\}, J=\{j\}, H=\{h\}$, then, $a_{i j}=a_{i h}$.

\subsection{Proof of Theorem 2.5}

We denote by $v \cdot x=\sum_{i j \in K_{n}-\mathrm{AW}_{p}^{k}} x_{i j} \leqslant p q$, the inequality (2.1). In this paragraph, we prove that $v \cdot x \leqslant p q$ defines a facet of the equicut polytope $\mathrm{EP}_{m}$ for all odd $m$ $m \geqslant n+2$ and $k \geqslant 1$. We set $m=n+r, r \geqslant 0$ even; hence, $m=2\left(q+k+\frac{1}{2} r\right)+1$ and, thus, the equicuts are the cuts $\delta(S)$ with $|S|=q+k+\frac{1}{2} r$ or $q+k+\frac{1}{2} r+1$. We denote by $\left[1^{\prime \prime}, r^{\prime \prime}\right]$ the $r$ additional nodes, so the complete graph $K_{m}$ has nodeset $[1, p] \cup$ $\left[1^{\prime}, q^{\prime}\right] \cup\left[1^{\prime \prime}, r^{\prime \prime}\right]$ and the equicut polytope $\mathrm{EP}_{m}$ is the polytope generated by the equicuts of $K_{m}$. Since $m$ is odd, $\mathrm{EP}_{m}$ is, in fact, the facet of the cut polytope $P_{m}$ induced by the inequality

$$
\sum_{i j \in K_{m}} x_{i j} \leqslant\left\lfloor\frac{1}{2} m\right\rfloor\left\lceil\frac{1}{2} m\right\rceil .
$$

We use the following characterization for proving that $v \cdot x \leqslant p q$ defines a facet of $\mathrm{EP}_{m}$. Let $a \cdot x \leqslant a_{0}$ be a valid inequality for $\mathrm{EP}_{m}$ such that $\left\{x \in \mathrm{EP}_{m}: v \cdot x=p q\right\} \subseteq$ $\left\{x \in \mathrm{EP}_{m}: a \cdot x=a_{0}\right\}$. We show that there exist some scalars $A>0, B$ such that: $a \cdot x=A v \cdot x+B\left(\sum_{1 \leqslant i<j \leqslant m} x_{i j}\right)$ and $a_{0}=A p q+B\left\lfloor\frac{1}{2} m\right\rfloor\left[\frac{1}{2} m\right\rceil$. It suffices, in fact, to show the existence of scalars $\alpha, \beta$ for which the following statements hold:

(a) $a_{i j}=\alpha$ for all $i j \in K_{n}-\mathrm{AW}_{p}^{k}$.

(b) $a_{i j}=\beta$ for all $i j \in \mathrm{AW}_{p}^{k}$.

(c) $a_{i^{\prime \prime} j^{\prime \prime}}=\beta$ for all $1^{\prime \prime} \leqslant i^{\prime \prime}<j^{\prime \prime} \leqslant r^{\prime \prime}$.

(d) $a_{i j^{\prime \prime}}=\beta$ for all $i \in[1, p] \cup\left[1^{\prime}, q^{\prime}\right], j^{\prime \prime} \in\left[1^{\prime \prime}, r^{\prime \prime}\right]$.

The roots in $\mathrm{EP}_{m}$ of the inequality $v \cdot x \leqslant p q$ are the cuts $\delta(S \cup T)$, where $S$ is a subset of $[1, p] \cup\left[1^{\prime}, q^{\prime}\right]$ such that $\delta(S)$ is a root in $P_{n}$ of the inequality $v \cdot x \leqslant p q$, and $T$ is an arbitrary subset of $\left[1^{\prime \prime}, r^{\prime \prime}\right]$ such that $|S \cup T|=q+k+\frac{1}{2} r$ or $q+k+\frac{1}{2}+1$. 
Hence, from Remark 2.4, the roots of the inequality $v \cdot x \leqslant p q$ in $\mathrm{EP}_{m}$ include the cuts $\delta(S)$ where $S$ is of the following form:

(1) $S=S_{+} \cup\left[1^{\prime}, q^{\prime}\right] \cup T$, where $S_{+}$is a subset of size $s_{+}$contained in an interval of $[1, p]$ of size $k+1, T$ is a subset of $\left[1^{\prime \prime}, r^{\prime \prime}\right]$ of size $t$ and $t+s_{+}=k+\frac{1}{2} r$ or $k+\frac{1}{2} r+1$.

(2) $S=S_{+} \cup S_{-} \cup T$, where $S_{+}$is an interval of $[1, p]$ of size $s_{+}, k+1 \leqslant s_{+} \leqslant p-k$,

$S_{-}$is a subset of $\left[1^{\prime}, q^{\prime}\right]$ of size $s_{-}, T$ is a subset of $\left[1^{\prime \prime}, r^{\prime \prime}\right]$ of size $t$ such that either, $s_{+}+s_{-}=q+k$ and $t=\frac{1}{2} r$ or $\frac{1}{2} r+1$, or, $s_{+}+s_{-}=q+k+1$ and $t=\frac{1}{2} r-1$ or $\frac{1}{2} r$.

Claim 3.3. For some scalar $\gamma, a_{i j^{\prime}}=\gamma$ for all $1 \leqslant i \leqslant p, 1^{\prime} \leqslant j^{\prime} \leqslant q^{\prime}$.

Proof. Let $T$ be a subset of size $\frac{1}{2} r$ of $\left[1^{\prime \prime}, r^{\prime \prime}\right]$. Take distinct nodes $i^{\prime}, j^{\prime}$ in $\left[1^{\prime}, q^{\prime}\right]$ and set $S=[2, k+2] \cup\left[1^{\prime}, q^{\prime}\right]-\left\{i^{\prime}, j^{\prime}\right\} \cup T$. The sets $S \cup\left\{i^{\prime}\right\}, S \cup\left\{j^{\prime}\right\}, S \cup\left\{1, i^{\prime}\right\}$ and $S \cup\left\{1, j^{\prime}\right\}$ define roots of $v \cdot x=p q$, hence of $a \cdot x=a_{0}$. Therefore, Lemma 3.2 implies that $a_{1 i^{\prime}}=a_{1 j^{\prime}}$, i.e., $a_{11^{\prime}}=\cdots=a_{1 q^{\prime}}=\gamma_{1}$, for some scalar $\gamma_{1}$. Similarly, $a_{i 1^{\prime}}=\cdots=$ $a_{i q^{\prime}}=\gamma_{i}$ for some scalar $\gamma_{i}$, for all $i$. Take now $i, k+2 \leqslant i \leqslant p-k$, and $S=[2, i-1] \cup$ $\left[(i-k)^{\prime}, q^{\prime}\right] \cup T$, setting $\left[(i-k)^{\prime}, q^{\prime}\right]=\emptyset$ for $i=p-k$. The sets $S \cup\{1\}, S \cup\{i\}, S \cup$ $\left\{1,1^{\prime}\right\}$ and $S \cup\left\{1^{\prime}, i\right\}$ are roots. Lemma 3.2 implies again that $a_{11^{\prime}}=a_{i 1^{\prime}}$, i.e., $\gamma_{1}=\gamma_{i}$. So, $\gamma_{1}=\gamma_{k+2}=\cdots=\gamma_{p-k}$; similarly, $\gamma_{2}=\gamma_{k+3}=\cdots=\gamma_{p-k+1}$ and therefore, $\gamma_{1}=\gamma_{2}$. Henceforth, by symmetry, $\gamma_{1}=\gamma_{2}=\cdots=\gamma_{p}=\gamma$.

Claim 3.4. For some scalar $\alpha, a_{i^{\prime} j^{\prime}}=\alpha$ for all $1^{\prime} \leqslant i^{\prime}<j^{\prime} \leqslant q^{\prime}$.

Proof. Let $T$ be a subset of $\left[1^{\prime \prime}, r^{\prime \prime}\right]$ of size $\frac{1}{2} r$. Take distinct nodes $i^{\prime}, j^{\prime}, h^{\prime}$ in $\left[1^{\prime}, q^{\prime}\right]$ and set $S=[1, k+2] \cup\left[1^{\prime}, q^{\prime}\right]-\left\{i^{\prime}, j^{\prime}, h^{\prime}\right\} \cup T$. The sets $S \cup\left\{j^{\prime}\right\}, S \cup\left\{h^{\prime}\right\}, S \cup\left\{i^{\prime}, j^{\prime}\right\}$ and $S \cup\left\{i^{\prime}, h^{\prime}\right\}$ all define roots; hence, we deduce from Lemma 3.2 that $a_{i^{\prime} j^{\prime}}=a_{i^{\prime} h^{\prime}}$, henceforth stating the result.

Claim 3.5. $\alpha=\gamma$.

Proof. Take $S=[2, k+2] \cup\left[3^{\prime}, q^{\prime}\right] \cup T$, where $T$ is a subset of $\left[1^{\prime \prime}, r^{\prime \prime}\right]$ of size $\frac{1}{2} r$. The sets $S \cup\{1\}, S \cup\left\{1^{\prime}\right\}, S \cup\left\{1^{\prime}, 2^{\prime}\right\}$ and $S \cup\left\{1,1^{\prime}\right\}$ define roots; thus, Lemma 3.2 implies that $a_{11^{\prime}}=a_{1^{\prime} 2^{\prime}}$, i.e., $\alpha=\gamma$.

Claim 3.6. $a_{i j}=\alpha$ for all $i j \in K_{n}-\mathrm{AW}_{p}^{k}$.

Proof. Take $u, k+2 \leqslant u \leqslant p-k$, and a subset $T$ of size $\frac{1}{2} r$ of $\left[1^{\prime \prime}, r^{\prime \prime}\right]$. Set $S=$ $[2, u-1] \cup\left[2^{\prime},(p-u+k+2)^{\prime}\right] \cup T$; then $S \cup\{u\}, S \cup\left\{1^{\prime}\right\}, S \cup\{1, u\}, S \cup\left\{1,1^{\prime}\right\}$ define roots, implying that $a_{1 u}=a_{11^{\prime}}=\alpha$ and the general result follows by symmetry.

Remark. At this point, we can already deduce that, in the case $k=1, v \cdot x \leqslant p q$ is facet inducing for $\mathrm{EP}_{n}$, i.e., with $r=0$; for this, the only remaining thing to check is that $a_{i, i+1}=\beta$ for all $1 \leqslant i \leqslant p$. This fact follows from Lemma 3.2 applied to the roots $\delta(S \cup\{1\}), \delta(S \cup\{3\}), \delta(S \cup\{1,2\})$ and $\delta(S \cup\{2,3\})$ with $S=\left[1^{\prime}, q^{\prime}\right]$, implying $a_{12}=a_{23}$. 
Remains to show that, for some scalar $\beta, a_{i j}=\beta$ for $(i, j) \in \mathrm{AW}_{p}^{k}$ and $a_{i^{\prime \prime} i}=a_{i^{\prime \prime} i^{\prime}}=$ $a_{i^{\prime \prime} j^{\prime \prime}}=\beta$ for $1 \leqslant i \leqslant p, 1^{\prime} \leqslant i^{\prime} \leqslant q^{\prime}$ and $1^{\prime \prime} \leqslant i^{\prime \prime}<j^{\prime \prime} \leqslant r^{\prime \prime}$.

If $i^{\prime \prime}, j^{\prime \prime}, h^{\prime \prime}$ are distinct nodes of $\left[1^{\prime \prime}, r^{\prime \prime}\right]$, let $T$ be a subset of $\left[1^{\prime \prime}, r^{\prime \prime}\right]-\left\{i^{\prime \prime}, j^{\prime \prime}, h^{\prime \prime}\right\}$ of size $\frac{1}{2} r-1$ and $S=[1, k] \cup\left[1^{\prime}, q^{\prime}\right] \cup T$, then $S \cup\left\{j^{\prime \prime}\right\}, S \cup\left\{h^{\prime \prime}\right\}, S \cup\left\{i^{\prime \prime}, j^{\prime \prime}\right\}$ and $S \cup\left\{i^{\prime \prime}, h^{\prime \prime}\right\}$ define roots which implies that $a_{i^{\prime \prime} j^{\prime \prime}}=a_{i^{\prime \prime} h^{\prime \prime}}$. Therefore, there exists a scalar $\beta$ such that $a_{i^{\prime \prime} j^{\prime \prime}}=\beta$ for all $1^{\prime \prime} \leqslant i^{\prime \prime}<j^{\prime \prime} \leqslant r^{\prime \prime}$.

Claim 3.7. $a_{i i^{\prime \prime}}=a_{i^{\prime} i^{\prime \prime}}=\beta$ for all $1 \leqslant i \leqslant p, 1^{\prime} \leqslant i^{\prime} \leqslant q^{\prime}, 1^{\prime \prime} \leqslant i^{\prime \prime} \leqslant r^{\prime \prime}$.

Proof. Take $i^{\prime \prime}, j^{\prime \prime}$ in $\left[1^{\prime \prime}, r^{\prime \prime}\right]$ and let $T$ be a subset of $\left[1^{\prime \prime}, r^{\prime \prime}\right]-\left\{i^{\prime \prime}, j^{\prime \prime}\right\}$ of size $\frac{1}{2} r-1$ (note that this requires that $r \geqslant 2$ ). Set $S=[2, k+1] \cup\left[1^{\prime}, q^{\prime}\right] \cup T$; then, the sets $S \cup\{1\}, S \cup\left\{j^{\prime \prime}\right\}, S \cup\left\{1, i^{\prime \prime}\right\}, S \cup\left\{i^{\prime \prime}, j^{\prime \prime}\right\}$ define roots, implying that $a_{1 i^{\prime \prime}}=a_{i^{\prime \prime} j^{\prime \prime}}=\beta$. Set now $S^{\prime}=[1, k+1] \cup\left[2^{\prime}, q^{\prime}\right] \cup T$; then, $S^{\prime} \cup\left\{1^{\prime}\right\}, S^{\prime} \cup\left\{j^{\prime \prime}\right\}, S^{\prime} \cup\left\{1^{\prime}, i^{\prime \prime}\right\}$ and $S^{\prime} \cup\left\{i^{\prime \prime}, j^{\prime \prime}\right\}$ define roots, yielding $a_{1^{\prime} i^{\prime \prime}}=a_{i^{\prime \prime} j^{\prime \prime}}=\beta$.

Claim 3.8. $a_{i j}=\beta$ for all $i j \in \mathrm{AW}_{p}^{k}$.

Proof. Let $i^{\prime \prime} \in\left[1^{\prime \prime}, r^{\prime \prime}\right]$ and $T$ be a subset of $\left[1^{\prime \prime}, r^{\prime \prime}\right]-\left\{i^{\prime \prime}\right\}$ of size $\frac{1}{2} r$. We prove that $a_{1 u}=\beta$ for $2 \leqslant u \leqslant k+1$. For $S=[2, u-1] \cup[u+1, k+1] \cup\left[1^{\prime}, q^{\prime}\right] \cup T$, the sets $S \cup$ $\{1\}, S \cup\{u\}, S \cup\left\{i^{\prime \prime}, 1\right\}$ and $S \cup\{1, u\}$ define roots, implying $a_{1 i^{\prime \prime}}=a_{1 u}=\beta$.

\subsection{Proof of Theorem 2.2}

Set $v=\mathrm{CW}_{n}^{k}(k, \ldots, k,-1, \ldots,-1)$, consisting of $p$ coefficients $k, q$ coefficients -1 with $p k-q=2 k+1, k \geqslant 1$. We assume that $p \geqslant 5$, hence $q \geqslant 3 k+1$. As was mentioned in Example $1.5, \mathrm{AW}_{p}^{k}(k, \ldots, k)$ is exactly the cycle $C=(1,2, \ldots, p)$ with weight $\frac{1}{2} k(k+1)$ on its edges. In order to prove that $v \cdot x \leqslant 0$ is facet inducing for $C_{n}$, we take a valid inequality $a \cdot x \leqslant 0$ for $C_{n}$ such that $a \cdot x=0$ whenever $v \cdot x=0$. We must show the existence of a scalar $\alpha$ such that $a=\alpha v$, which amounts to checking that the following conditions hold:

(a) $a_{i, i+1}=\frac{1}{2} k(k-1) \alpha$ for all $1 \leqslant i \leqslant p$.

(b) $a_{i j}=k^{2} \alpha$ for all $i j \in K_{p}-C$.

(c) $a_{i^{\prime} j^{\prime}}=\alpha$ for all $i^{\prime} j^{\prime} \in K_{q}$.

(d) $a_{i j^{\prime}}=-k \alpha$ for all $1 \leqslant i \leqslant p, 1^{\prime} \leqslant j^{\prime} \leqslant q^{\prime}$.

Recall that, from Proposition 1.19, the roots of $v \cdot x \leqslant 0$ are the cuts $\delta(S)$ for which $S=S_{+} \cup S_{-}$where $S_{+}$is an interval of $[1, p]$ of size $s_{+}, S_{-}$is a subset of $\left[1^{\prime}, q^{\prime}\right]$ of size $s_{-}$and $s_{-}=k s_{+}-k$ or $k s_{+}-k-1$. The proof is given through the following claims.

Claim 3.9. For some scalar $\alpha, a_{i^{\prime} j^{\prime}}=\alpha$ for all $1^{\prime} \leqslant i^{\prime}<j^{\prime} \leqslant q^{\prime}$.

Proof. Take three distinct nodes $i^{\prime}, j^{\prime}, h^{\prime}$ in $\left[1^{\prime}, q^{\prime}\right]$, let $T$ be a subset of $\left[1^{\prime}, q^{\prime}\right]$ $\left\{i^{\prime}, j^{\prime}, h^{\prime}\right\}$ of size $k-2$ and set $S=T \cup\{1,2\}$. Then, the sets $S \cup\left\{j^{\prime}\right\}, S \cup\left\{h^{\prime}\right\}$, $S \cup\left\{i^{\prime}, j^{\prime}\right\}$ and $S \cup\left\{i^{\prime}, h^{\prime}\right\}$ all define roots which, from Lemma 3.2, implies that $a_{i^{\prime} j^{\prime}}=a_{i^{\prime} h^{\prime}}$, henceforth stating the result. 
Claim 3.10. For some scalar $\gamma, a_{i j^{\prime}}=\gamma$ for all $1 \leqslant i \leqslant p, 1^{\prime} \leqslant j^{\prime} \leqslant q^{\prime}$.

Proof. Take $i, j, h$ in $[1, p], i^{\prime}$ in $\left[1^{\prime}, q^{\prime}\right]$, let $T$ be a subset of $\left[1^{\prime}, q^{\prime}\right]-\left\{i^{\prime}\right\}$ of size $k-1$ and set $S=\{h\} \cup T$. The sets $S \cup\{i\}, S \cup\{j\}, S \cup\left\{i^{\prime}, i\right\}$ and $S \cup\left\{i^{\prime}, j^{\prime}\right\}$ all define roots, henceforth implying that $a_{i^{\prime} i}=a_{i^{\prime} j}$. Therefore, $a_{i^{\prime} 1}=\cdots=a_{i^{\prime} p}=\gamma_{i^{\prime}}$ for all $i^{\prime}$. Let $A, B$ be two disjoint subsets of $\left[1^{\prime}, q^{\prime}\right]-\left\{1^{\prime}, 2^{\prime}\right\}$ having both size $k-1$; we set $S=\{2,3\} \cup A$ and $I=\{1\} \cup B$. The sets $S \cup\left\{1^{\prime}\right\}, S \cup\left\{2^{\prime}\right\}, S \cup I \cup\left\{1^{\prime}\right\}$ and $S \cup I \cup\left\{2^{\prime}\right\}$ all define roots which, from Lemma 3.2 implies that $\sum_{i \in I} a_{i 1^{\prime}}=\sum_{i \in I} a_{i 2^{\prime}}$; using Claim 3.9 , we deduce that $a_{11^{\prime}}=a_{12^{\prime}}$. Hence $\gamma_{1^{\prime}}=\gamma_{2^{\prime}}$, and, by symmetry, $\gamma_{1^{\prime}}=\cdots=\gamma_{q^{\prime}}=$ $\gamma$.

Since each $\delta(\{i\})$ is root for $1 \leqslant i \leqslant p, a \cdot \delta(\{i\})=0$ holds, yielding the following relation:

$\left(\mathrm{S}_{i}\right) \quad \sum_{1 \leqslant j \leqslant p} a_{i j}=-\gamma q$.

Claim 3.11. $\gamma=-k \alpha$.

Proof. Since $\{1,2\} \cup\left[1^{\prime}, k^{\prime}\right]$ defines a root, we deduce the relation:

$$
0=\sum_{1 \leqslant j \leqslant p} a_{1 j}+a_{2 j}-2 a_{12}+2 \gamma(q-k)+k(p-2) \gamma+k(q-k) \alpha,
$$

which, using $\left(\mathrm{S}_{1}\right),\left(\mathrm{S}_{2}\right)$, implies: $2 a_{12}=-2 \gamma q+2 \gamma(q-k)+k(p-2) \gamma+k(q-k) \alpha$, i.e.,

(*) $2 a_{12}=\gamma k(p-4)+\alpha k(q-k)$.

Now, the set $\{1,2\} \cup\left[1^{\prime},(k-1)^{\prime}\right]$ also defines a root and, similarly, we obtain:

(**) $2 a_{12}=\gamma(k-1)(p-4)+\alpha(k-1)(q-k+1)$.

Therefore, substracting $(* *)$ from $(*)$ yields that: $0=\gamma(p-4)+\alpha(q-2 k+1)=$ $(p-4)(\gamma+\alpha k)$, using the fact that: $p-k q=2 k+1$. Since $p \geqslant 5$, we deduce that $\gamma=-\alpha k$. Then, relation (*) implies that $a_{12}=\frac{1}{2} \alpha k(k-1)$ which, by symmetry, states the next Claim 3.12.

Claim 3.12. $a_{i, i+1}=\frac{1}{2} \alpha k(k-1)$ for all $1 \leqslant i \leqslant p$.

Claim 3.13. $a_{13}=k^{2} \alpha$.

Proof. Using the fact that $\{1,2,3\} \cup\left[1^{\prime},(2 k)^{\prime}\right]$ defines a root and relations $\left(S_{i}\right)$, we deduce that: $0=3 k q \alpha-2\left(a_{12}+a_{13}+a_{23}\right)-3 k \alpha(q-2 k)-2 k^{2} \alpha(p-3)+2 k \alpha(q-2 k)$ and thus, using Claim 3.12, $a_{13}=k^{2} \alpha$.

We conclude the proof by showing, by induction on $u, 3 \leqslant u \leqslant \frac{1}{2}(p+1)$, the following assertion:

$\left(\mathrm{H}_{u}\right) \quad a_{i j}=k^{2} \alpha$ for all $i j \in K_{p}-C$.

For $u=3,\left(H_{u}\right)$ holds from Claim 3.13. Take $u \geqslant 4$ and assume that $\left(H_{i}\right)$ holds for $3 \leqslant i \leqslant u-1$; we prove in the next claim that $\left(\mathrm{H}_{u}\right)$ holds, i.e., $a_{1 u}=\cdots=a_{u-2, u}=k^{2} \alpha$. 
Claim 3.14. $a_{\mathrm{iu}}=k^{2} \alpha$ for all $1 \leqslant i \leqslant u-2$.

Proof. Both sets $S=[i, u] \cup\left[1^{\prime},((u-i) k)^{\prime}\right]$ and $S^{\prime}=[i+1, u] \cup\left[1^{\prime},((u-i-1) k)^{\prime}\right]$ define roots, yielding the relations

$$
\begin{aligned}
0=a \cdot \delta(S)= & \alpha k q(u-i+1)-2\left(\sum_{i \leqslant h<1 \leqslant u} a_{h l}\right)-k \alpha(u-i+1)(q-k(u-i)) \\
& -k \alpha(u-i) k(p-u+i-1)+\alpha(u-i) k(q-k(u-i))
\end{aligned}
$$

and

$$
\begin{aligned}
0=a \cdot \delta\left(S^{\prime}\right)= & \alpha k q(u-i)-2\left(\sum_{i+1 \leqslant h<1 \leqslant u} a_{h l}\right)-k \alpha(u-i)(q-k(u-i-1)) \\
& -k \alpha(u-i-1) k(p-u+i)+\alpha k(u-i-1)(q-k(u-i-1)) .
\end{aligned}
$$

Hence, using the induction assumption, $a_{i, i+1}=a_{i, i+2}=\cdots=a_{i, u-1}=k^{2} \alpha$ and the fact that $2 a_{i, i+1}=\alpha k(k-1)$, we deduce that

$$
\begin{aligned}
0= & a \cdot \delta(S)-a \cdot \delta\left(S^{\prime}\right) \\
= & \alpha k q-2 a_{i u}-\alpha k(k-1)-2 \alpha k^{2}(u-i-2)-k \alpha(q-2 k(u-i)) \\
& -\alpha k^{2}(p-2(u-i))+\alpha k(q+k-2 k(u-i)),
\end{aligned}
$$

implying that $a_{i u}=\alpha k^{2}$.

\subsection{Proof of Theorem 2.3}

Set $v=\mathrm{CW}_{n}^{k}(2,1, \ldots, 1,-1, \ldots,-1)$, consisting of $p-1$ coefficients $1, q$ coefficients $-1, p-q=2 k, k \geqslant 1$. We assume that $p \geqslant 2 k+3$, i.e., $q \geqslant 3$. We set $X=\{(1, k+1)$, $(1, p-k+1)\} \cup\{(p-i, k-i): i=0,1, \ldots, k-2\}$. From Example 1.6, we have that

$$
\begin{array}{ll}
v_{i j}=1 & \text { for } i j \in X, \\
v_{i j}=0 & \text { for } i j \in \mathrm{AW}_{p}^{k}-X, \\
v_{i j}=1 & \text { for } i j \in K_{p}-\mathrm{AW}_{p}^{k} \text { and } 2 \leqslant i \leqslant p, \\
v_{1 j}=2 & \text { for } k+2 \leqslant j \leqslant p-k .
\end{array}
$$

Let $a \cdot x \leqslant 0$ be a valid inequality for $C_{n}$ such that $a \cdot x=0$ whenever $v \cdot x=0$. We prove the existence of a scalar $\alpha$ such that $a=\alpha v$, i.e., for which the following statements hold:

(a) $a_{i j}=0$ for $i j \in \mathrm{AW}_{p}^{k}-X$,

(b) $a_{i j}=\alpha$ for $i j \in X \cup K_{p}-\mathrm{AW}_{p}^{k}$,

(c) $a_{1 j}=2 \alpha$ for $k+2 \leqslant j \leqslant p-k$,

(d) $a_{i j^{\prime}}=-\alpha$ for $2 \leqslant i \leqslant p, 1^{\prime} \leqslant j^{\prime} \leqslant q^{\prime}$,

(e) $a_{1 j^{\prime}}=-2 \alpha$ for $1^{\prime} \leqslant j^{\prime} \leqslant q^{\prime}$,

(f) $a_{i^{\prime} j^{\prime}}=\alpha$ for $1^{\prime} \leqslant i^{\prime}<j^{\prime} \leqslant q^{\prime}$.

We use the description of the roots given in Proposition 1.18. 
Claim 3.15. For some scalar $\alpha, a_{i^{\prime} j^{\prime}}=\alpha$ for $1^{\prime} \leqslant i^{\prime}<j^{\prime} \leqslant q^{\prime}$.

Proof. The sets $S \cup\left\{j^{\prime}\right\}, S \cup\left\{h^{\prime}\right\}, S \cup\left\{i^{\prime}, j^{\prime}\right\}$ and $S \cup\left\{i^{\prime}, h^{\prime}\right\}$, where $S=[2, k+3]$ and $i^{\prime}, j^{\prime}, h^{\prime}$ are nodes of $\left[1^{\prime}, q^{\prime}\right]$, define roots which implies that $a_{i^{\prime} j^{\prime}}=a_{i^{\prime} h^{\prime}}$, hence stating the result.

Claim 3.16. For some scalars $\gamma, \gamma_{1}, a_{1 i^{\prime}}=\gamma_{1}$ and $a_{i i^{\prime}}=\gamma$ for $2 \leqslant i \leqslant p, 1^{\prime} \leqslant i^{\prime} \leqslant q^{\prime}$.

Proof. For $i^{\prime}, j^{\prime}$ in $\left[1^{\prime}, q^{\prime}\right]$ and $S=[3, k+3]$, the sets $S \cup\left\{i^{\prime}\right\}, S \cup\left\{j^{\prime}\right\}, S \cup\left\{2, i^{\prime}\right\}$ and $S \cup\left\{2, j^{\prime}\right\}$ define roots, implying $a_{2 i^{\prime}}=a_{2 j^{\prime}}=\gamma_{2}$; similarly, $a_{i j^{\prime}}=y_{i}$ for all $i \geqslant 2, j^{\prime}$. For $i, j$ in $[1, p]$ with $i \geqslant 2, i+k+1 \leqslant j \leqslant i+p-k$, and $S^{\prime}=[i+1, j-1] \cup$ $\left[2^{\prime},(j-i-k)^{\prime}\right]$, the sets $S^{\prime} \cup\{i\}, S^{\prime} \cup\{j\}, S^{\prime} \cup\left\{1^{\prime}, i\right\}$ and $S^{\prime} \cup\left\{1^{\prime}, j\right\}$ define roots; hence $a_{1^{\prime} i}=a_{1^{\prime} j}$ from which we deduce $\gamma_{2}=\cdots=\gamma_{p}$. Now, let $h^{\prime}$ be a node in $\left[1^{\prime}, q^{\prime}\right]$ distinct from $i^{\prime}, j^{\prime}$ (this is possible since, by assumption, $q \geqslant 3$ ), $S^{\prime \prime}=[2, k+2]$ and $A=\left\{1, h^{\prime}\right\}$; then $S^{\prime \prime} \cup\left\{i^{\prime}\right\}, S^{\prime \prime} \cup\left\{j^{\prime}\right\}, S^{\prime \prime} \cup A \cup\left\{i^{\prime}\right\}$ and $S^{\prime \prime} \cup A \cup\left\{j^{\prime \prime}\right\}$ define roots, which, from Lemma 3.2, yields that: $a_{1 i^{\prime}}+a_{i^{\prime} h^{\prime}}=a_{1 j^{\prime}}+a_{j^{\prime} h^{\prime}}$, i.e., using Claim 3.15, $a_{1 i^{\prime}}=a_{1 j^{\prime}}=\gamma_{1}$.

Since $\delta(\{i\})$ is a root for all $1 \leqslant i \leqslant p$, we deduce the following relations:

$$
\sum_{1 \leqslant j \leqslant p} a_{1 j}=-q \gamma_{1}
$$

$$
\sum_{1 \leqslant j \leqslant p} a_{i j}=-q \gamma \text { for } 2 \leqslant i \leqslant p .
$$

Claim 3.17. $\gamma=-\alpha$ and $\gamma_{1}=-2 \alpha$.

Proof. Both sets $S=[2, k+2]$ and $S \cup\left\{1^{\prime}\right\}$ define roots, hence $0=$ $a \cdot \delta\left(S \cup\left\{1^{\prime}\right\}\right)-a \cdot \delta(S)$, implying

(*) $\quad 0=\gamma_{1}+\gamma(p-2 k-3)+\alpha(q-1)$.

Similarly, since both sets $S^{\prime}=[1, k]$ and $S^{\prime} \cup\left\{1^{\prime}\right\}$ define roots, we deduce

$$
\text { (**) } \quad 0=-\gamma_{1}+\gamma(p-2 k+1)+\alpha(q-1) \text {. }
$$

Substracting $(* *)$ from $(*)$ yields $0=(2 \gamma+2 \alpha)(p-2 k-1)$, hence $\gamma=-\alpha$ from which we deduce that $\gamma_{1}=-2 \alpha$.

Claim 3.18. $a_{i j}=0$ for all $i j \in \mathrm{AW}_{p}^{k}-X$.

Proof. Take $i, u, 1 \leqslant u \leqslant k, 2 \leqslant i \leqslant p-u$ and $S=[i+1, i+u-1]$; since $S, S \cup\{i\}$, $S \cup\{i+u\}$ and $S \cup\{i, i+u\}$ define roots, we deduce from Lemma 3.1 that $a_{i, i+u}=0$. Remains to show that $a_{p-k+i, j}=0$ for $2 \leqslant i \leqslant k, 1 \leqslant j \leqslant i-1$; for this, set $S^{\prime}=$ $[p-k+i+1, j-1]$ (containing node 1) and note that $S, S \cup\{j\}, S \cup\{p-k+i\}$ and $S \cup\{j, p-k+i\}$ define roots, which gives the result. 
Claim 3.19. $a_{i j}=\alpha$ for all $i j \in X$.

Proof. Using the fact that both sets $[1, k] \cup\left\{1^{\prime}\right\}$ and $[1, k+1] \cup\left\{1^{\prime}\right\}$ define roots, we deduce

$$
0=\sum a_{i, k+1}-2\left(a_{1, k+1}+\cdots+a_{k, k+1}\right)+\gamma(q-1)-\gamma,
$$

which, from the above claims, implies that $a_{1, k+1}=\alpha$; similarly, $a_{1, p-k+1}=\alpha$. From the fact that $[1, k] \cup\left\{1^{\prime}\right\}$ and $[1, k] \cup\left\{p, 1^{\prime}\right\}$ define roots, we obtain

$$
0=\sum a_{p i}-2\left(a_{1 p}+\cdots+a_{k p}\right)+\gamma(q-2),
$$

implying $a_{p k}=\alpha$; similarly, $a_{p-i, k-i}=\alpha$ for $1 \leqslant i \leqslant k-2$.

Claim 3.20. $a_{1, k+2}=2 \alpha$.

Proof. For establishing that $a_{1, k+2}=2 \alpha$, see that $S=[1, k+2] \cup\left\{1^{\prime}, 2^{\prime}\right\}$ defines a root and use relation: $0=a \cdot \delta(S)$.

We finish the proof by showing, by induction on $u, k+2 \leqslant u \leqslant \frac{1}{2}(p+1)$, that the following relation $\left(\mathrm{H}_{u}\right)$ holds:

$\left(\mathrm{H}_{u}\right) \quad a_{1 j}=2 \alpha$ for $k+2 \leqslant j \leqslant u$ and $a_{i j}=\alpha$ for $2 \leqslant i, i+k+1 \leqslant j \leqslant u$.

Relation $\left(\mathrm{H}_{k+2}\right)$ holds from Claim 3.20. Assuming that $\left(\mathrm{H}_{w}\right)$ holds for $w<u$, we prove that $\left(\mathrm{H}_{u}\right)$ holds, i.e., we compute $a_{1 u}, a_{2 u}, \ldots, a_{u-k, u}$. From the fact that $[1, u] \cup\left[1^{\prime},(u-k)^{\prime}\right]$ defines a root, we deduce

(*) $\quad 0=-2\left(\sum_{1 \leqslant i<j \leqslant u} a_{i j}\right)+\alpha(u-k)(1+u-k)$.

From the fact that $[2, u] \cup\left[1^{\prime},(u-k-1)^{\prime}\right]$ defines a root, we deduce

$$
\text { (**) } \quad 0=-2\left(\sum_{2 \leqslant i<j \leqslant u} a_{i j}\right)+\alpha(u-k-1)(u-k-2) .
$$

Substracting (**) from (*) yields that $a_{1 u}=2 \alpha$. Finally, take $2 \leqslant w \leqslant u-k-1$ and $S=[w+1, u] \cup\left[1^{\prime},(u-w-k)^{\prime}\right]$; since both $S$ and $S \cup\{w\}$ define roots, we have $0=a \cdot \delta(S \cup\{w\})-a \cdot \delta(S)$, from which we obtain that $a_{w u}=2 \alpha$.

\section{Acknowledgements}

We thank the referees for their suggestions which helped to improve the presentation of the paper. We also thank Cid De Souza for pointing out an error in an earlier formulation of Theorem 1.8. 


\section{References}

[1] N. Alon, "The CW-inequalities for vectors in $l_{1}$," European Journal of Combinatorics 11 (1990) 1-6.

[2] P. Assouad and M. Deza, "Espaces métriques plongeables dans un hypercube: aspects combinatoires," Annals of Discrete Mathematics 8 (1980) 197-210.

[3] P. Assouad and M. Deza, "Metric subspaces of $L_{1}$ " Publications mathématiques d'Orsay 3 (1982).

[4] D. Avis and M. Deza, "The cut cone, $L^{1}$-embeddability, complexity and multicommodity flows," Networks 21 (1991) 595-617.

[5] F. Barahona, M. Grötschel, M. Jünger and G. Reinelt, "An application of combinatorial optimization to statistical physics and circuit layout design," Operations Research 36(3) (1988) 493-513.

[6] F. Barahona, M. Grötschel and A.R. Mahjoub, "Facets of the bipartite subgraph polytope," Mathematics of Operations Research 10 (1985) 340 358.

[7] F. Barahona and A.R. Mahjoub, "On the cut polytope," Mathematical Programming 36 (1986) 157-173.

[8] M. Conforti, M.R. Rao and A. Sassano, "The equipartition polytope I,II," Mathematical Programming 49 (1990) 49-70, 71-91.

[9] C. De Simone, M. Deza and M. Laurent, "Collapsing and lifting for the cut cone," to appear in: Proceedings of the Second Japan Conference on Graph Theory and Combinatorics (Hakone, 1990).

[10] M. Deza, "On the Hamming geometry of unitary cubes," Doklady Adademii Nauk SSR 134 (1960) 1037-1040. [English translation in: Soviet Physics Doklady 5 (1961) 940-943.]

[11] M. Deza, "Matrices de formes quadratiques non négatives pour des arguments binaires," Comptes Rendus de l'Acadêmie des Sciences de Paris 277 (1973) 873-875.

[12] M. Deza, K. Fukuda and M. Laurent, "The inequicut cone," Research Report No. 89-04, GSSM, University of Tsukuba (Tokyo, 1989).

[13] M. Deza, V.P. Grishukhin and M. Laurent, "The symmetries of the cut polytope and of some relatives," P. Gritzmann and B. Sturmfels, eds., Applied Geometry and Discrete Mathematics, The "Victor Klee Festschrift", DIMACS series in Discrete Mathematics and Theoretical Computer Sciences 4 (1991) 205-220.

[14] M. Deza, V.P. Grishukhin and M. Laurent, "The hypermetric cone is polyhedral," to appear in: Combinatorica.

[15] M. Deza, M. Grötschel and M. Laurent, "Clique-web facets for multicut polytopes," to appear in: Mathematics of Operations Research.

[16] M. Deza and M. Laurent, "Facets for the cut cone 1," Mathematical Programming 56(1992) 121-160, this issue.

[17] M. Deza and M. Laurent, "New results on facets of the cut cone," to appear in: R.C. Bose Memorial Issue, Joumal of Combinatorics, Information and System Sciences (1992).

[18] J.B. Kelly, "Hypermetric spaces" Lectures Notes in Mathematics No. 490 (Springer, Berlin, 1975) pp. $17-31$. 